ENCYCLOPÉDIE Encyclopédie berbère

BERBERE

5 | 1988

5 | Anacutas - Anti-Atlas

\title{
Anthropologie (Partie II)
}

M.-C. Chamla et D. Ferembach

\section{OpenEdition}

Journals

Édition électronique

URL : http://journals.openedition.org/encyclopedieberbere/2896

DOI : 10.4000/encyclopedieberbere.2896

ISSN : 2262-7197

\section{Éditeur}

Peeters Publishers

\section{Édition imprimée}

Date de publication : 1 avril 1988

Pagination : 713-775

ISBN : 2-85744-379-6

ISSN : 1015-7344

\section{Référence électronique}

M.-C. Chamla et D. Ferembach, « Anthropologie (Partie II) », Encyclopédie berbère [En ligne], 5| 1988, document A234, mis en ligne le 01 décembre 2012, consulté le 13 octobre 2020. URL : http://

journals.openedition.org/encyclopedieberbere/2896; DOI : https://doi.org/10.4000/ encyclopedieberbere.2896

Ce document a été généré automatiquement le 13 octobre 2020

(c) Tous droits réservés 


\title{
Anthropologie (Partie II)
}

\author{
M.-C. Chamla et D. Ferembach
}

\section{Anthropologie (Protohistoire et Antiquité) (M.- C. Chamla)}

1 Dès le début de la conquête de l'Algérie, la découverte des monuments mégalithiques que l'on y rencontre par dizaines de milliers suscita l'intérêt des archéologues et des anthropologues, sur cette époque que l'on dénomma, pendant longtemps, préislamique, mais qu'il est d'usage actuellement d'appeler protohistorique à la suite de G. Camps qui leur consacra en 1961 un ouvrage fondamental.

2 Les ensembles mégalithiques d'Afrique du nord constituent un groupe original qu'on ne peut rattacher au grand ensemble mégalithique de l'Europe occidentale, bien que parmi eux, les dolmens, dans leurs formes littorales - différentes de celle de l'intérieur des terres -, s'apparentent aux dolmens des pays méditerranéens, du Languedoc, de Sardaigne et de l'Italie péninsulaire.

3 Une classification de leurs formes, qui sont variées et nombreuses ainsi que leur répartition, a été proposée par Camps en 1961, 1963 et 1965. Parmi les plus courantes, les dolmens sont très nombreux en Algérie orientale et en Tunisie centrale, mais deviennent plus rares en Algérie centrale où ils se cantonnent dans la région littorale et sont pratiquement absents en Oranie et au Maroc. Vers le sud, ils ne dépassent pas l'Atlas saharien. Les nécropoles les plus méridionales se rencontrent dans la région de Tébessa, dans l'Aurès, et, plus à l'ouest, dans la région de Djelfa. En Tunisie, la répartition des haouanet (hypogées), autre forme très répandue de sépultures avec les dolmens, est plus limitée. Ils se concentrent dans le nord et le nord-est de la Tunisie. Tandis qu'au Maroc et en Algérie occidentale, ce sont les formes à tumuli qui prédominent.

4 Alors qu'au Maroc et en Algérie occidentale on trouve des traces indéniables de trait culturels ibériques dans ces sépultures (métallurgie, vases campaniformes, tombes en forme de ciste et de silo), à l'est, en Algérie orientale et en Tunisie, les sépultures mégalithiques et hypogées paraissent avoir une origine méditerranéenne centrale et 
orientale. Le matériel récolté, en particulier la céramique modelée, offre notamment des affinités remarquables avec les poteries italico-sicules, tant en ce qui concerne les formes que le décor (Camps, 1959 et 1974).

5 La civilisation mégalithique d'Afrique du Nord est certainement moins ancienne que celle d'Europe. Celle-ci existait dans le sud de l'Espagne et de l'Italie à la fin du $\mathrm{III}^{\mathrm{e}}$ et durant le II $^{e}$ millénaire. On ignore la date de son introduction en Afrique du nord. La datation des monuments nord-africains rencontre en effet des difficultés en raison, d'une part de la pauvreté du mobilier funéraire qui ne permet pas de fixer des repères chronologiques, mais aussi de la variété des objets provenant d'époques diverses qu'on y trouve parfois, même dans des sépultures qui n'avaient pas encore été dévastées comme beaucoup l'avaient été - avant leur fouille systématique.

6 Les restes trouvés dans les sépultures protohistoriques sont plus ou moins contemporains de ceux trouvés en Tunisie dans les sépultures d'époque punique. L'époque punique, qui se situe entre le $\mathrm{IX}^{\mathrm{e}}$ siècle et le $\mathrm{II}^{\mathrm{e}}$ siècle av. J.-C., doit être incluse dans la protohistoire du Maghreb (Camps, 1970). La culture mégalithique, néanmoins, paraît être arrivée au Maghreb avant celle des Puniques, elle y a subsisté pendant longtemps, comme le montrent les dates obtenues d'après le mobilier et les restes osseux de certaines sépultures, après la fin de l'époque punique, jusqu'au début de l'époque romaine.

Crânes d'époque protohistorique : 1) Crâne masculin de Tiddis $n^{\circ} 96$; 2) Crâne masculin négroïde de Gastel $n^{\circ} 10$; 3) Crâne masculin de type Méchtoïde de Sila.

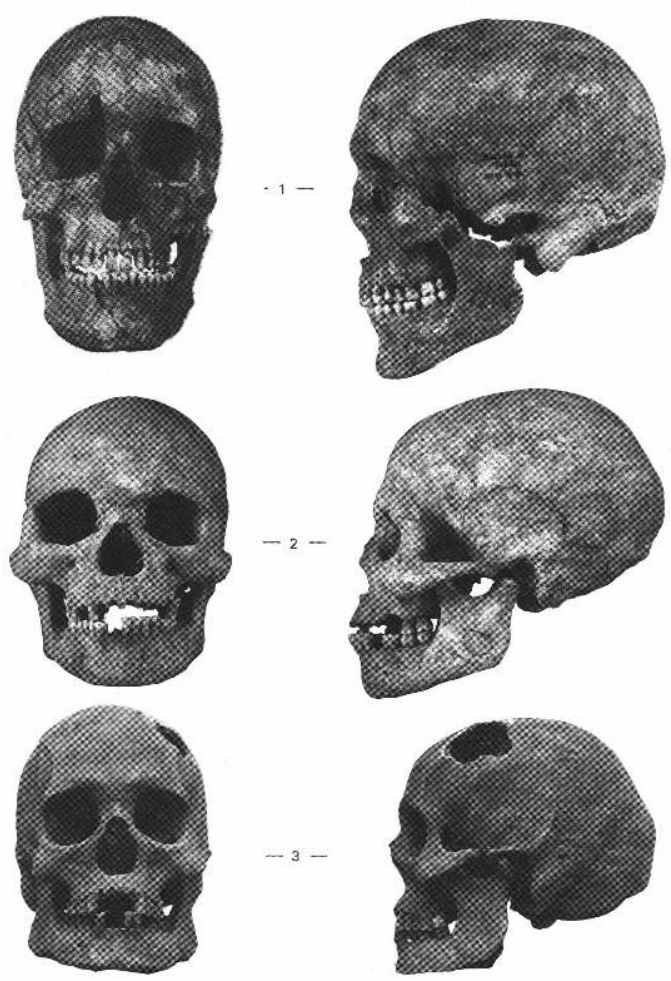

7 Les restes osseux qui ont été recueillis dans les sépultures protohistoriques sont beaucoup moins nombreux que ces dernières. En dehors du fait qu'elles ne contenaient pas toutes, tant s'en faut, de restes humains, ceux-ci ne nous sont pas parvenus en très grand nombre ni en très bon état, soit que l'état du sol n'ait pas permis leur 
conservation, qu'ils aient été dispersés en raison de la pratique fréquente des inhumations secondaires ou des réutilisations de sépultures, qu'ils aient été détruits par incinération, ou bien qu'ils aient été perdus après leur découverte souvent ancienne.

Les inhumations définitives ou primaires montrent une position des corps en décutibus latéral fléchi ou contracté fréquent, ou en décubitus dorsal ou latéral étendu, pratique plus rare et tardive, introduite par les Phéniciens puis les Romains. Les trois positions, néanmoins, peuvent être rencontrées dans une même nécropole qui indiquent la juxtaposition et la contemporanéité de rites différents (Camps, 1961). Dans les inhumations secondaires, les ossements sont soit mélangés et repoussés le long des parois (Sigus, Bou Merzoug), répandus sur la surface de la fosse (Bou Nouara), ou rangés dans un certain ordre (Dougga, Sila, Tiddis).

Les études anthropologiques qui leur ont été consacrées sont pour la plupart anciennes et fragmentaires. Citons celles de Faidherbe $(1867,1872)$ et de Pruner-Bey $(1869)$ sur les crânes de la nécropole de Roknia; de Bourjot (in Bertherand, 1868) sur ceux des dolmens de Beni Messous; de Letourneau et Papillault sur ceux du Medracen (1869); Bertholon et Chantre tentèrent de faire une synthèse sur les « Africains des sépultures mégalithiques » en 1913, mais les résultats en sont peu utilisables. Plus récemment, nous avons la brève étude de Leblanc sur quelques crânes de trois nécropoles d'Algérie centrale (in Roffo, 1938), celle de Marchand sur les crânes de Beni Messous (1930 et 1951). Enfin, nous devons une étude pathologique des crânes provenant de diverses sépultures d'Algérie à J. Dastugue (1973).

Des restes d'époque punique découverts dans les tombeaux de Carthage et d'Utique, dans les sépultures en jarre, dans les tombes à puits ou ceux trouvés dans les tombes à toit à deux pans, et peut-être plus anciens, ont fait l'objet d'études principalement de Bertholon en 1890, 1911 et 1913, de Hamy (in Quatrefages et Hamy, 1882) et de Collignon (1892).

11 Dans l'ensemble, ces publications n'offrent pas de caractère suffisamment synthétique pour que puisse se dégager une idée très précise sur la morphologie de la population de l'époque protohistorique d'Afrique du nord. Des fouilles récentes ayant permis la découverte de nouveaux restes venant s'ajouter aux séries déposées dans les collections du musée du Bardo à Alger et du musée de l'Homme principalement, il est possible d'envisager une étude d'ensemble sur la population de cette époque actuellement assez bien connue du point de vue archéologique.

Les données archéologiques ont montré l'introduction de traits culturels tout à fait nouveaux en Afrique du nord avec les sépultures mégalithiques qui offrent des affinités avec le monde méditerranéen occidental et central. Décèle-t-on également des affinités sur le plan anthropologique entre Protohistoriques et Puniques d'Afrique du nord et d'autres populations du Bassin méditerranéen vivant à la même époque ? Ces affinités, si elles existent, sont-elles dues à l'arrivée d'éléments nouveaux suffisamment importants pour modifier la composition de la population? On tentera de répondre à la première question dans la présente étude, la seconde ne pouvant être abordée qu'au moyen d'une analyse diachronique des restes humains des époques précédentes.

13 Les restes humains étudiés ici proviennent des collections du Centre de recherches anthropologiques, préhistoriques et ethnographiques d'Alger, du musée archéologique de Constantine et du laboratoire d'Anthropologie du musée de l'Homme de Paris. 
14 En nous basant sur les restes crâniens entiers, les fragments de crânes et les mandibules isolées, nous avons dénombré 241 individus se répartissant en 125 hommes, 96 femmes, 17 enfants et 3 sujets de sexe non définissable. Le tableau I indique leur répartition et leur origine géographique.

Localisation des sites protohistoriques et puniques cités dans le texte.

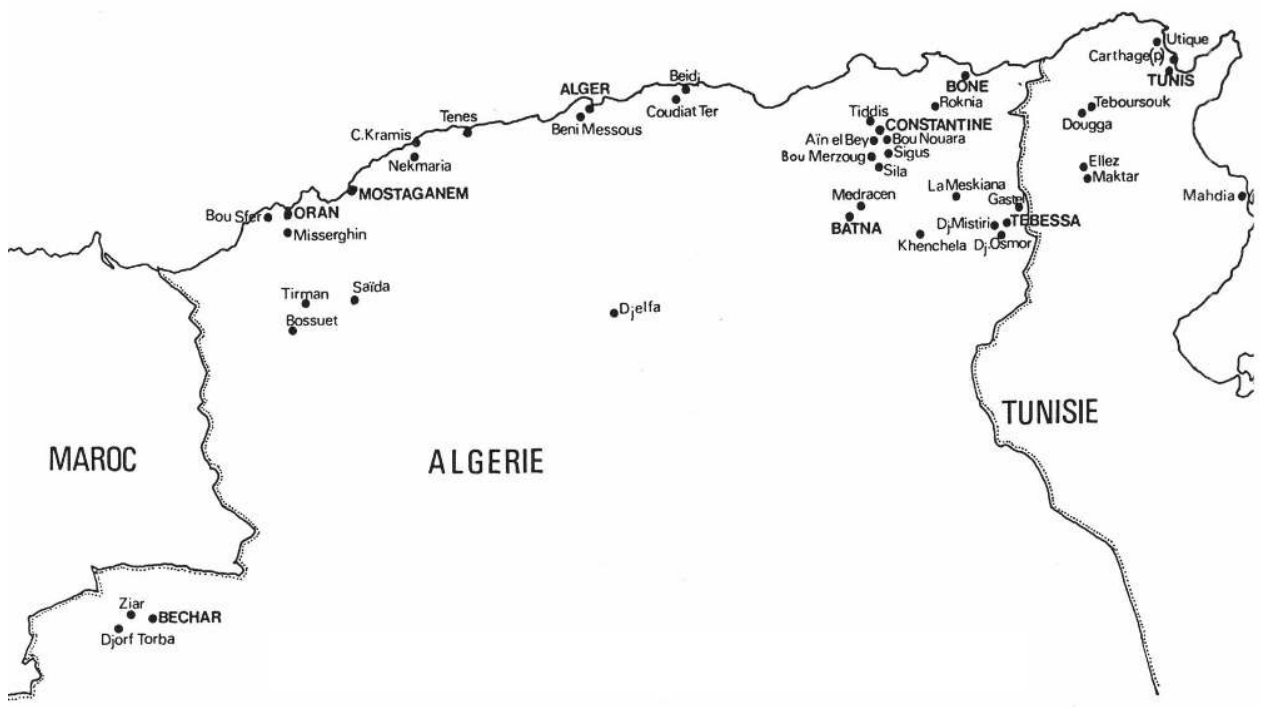

Parmi ces restes inégalement conservés, il nous a été possible d'étudier 70 crânes masculins et 54 crânes féminins algériens, 19 crânes masculins et 18 crânes féminins d'époque punique. Les séries d'Algérie occidentale et centrale étant peu représentées, nous avons constitué trois séries d'importance inégale: une de protohistoriques d'Algérie occidentale et centrale, une de protohistoriques d'Algérie orientale, une troisième série regroupant les quelques protohistoriques et les puniques de Tunisie. 
Tableau 1. - Origine des sujets.

\begin{tabular}{|c|c|c|c|c|}
\hline & Hommes & Femmes & Enfants & $\begin{array}{c}\text { Sexe } \\
\text { non } \\
\text { déterminé }\end{array}$ \\
\hline $\begin{array}{l}\text { I. Algérie occidentale (sépult. protohist.) : } \\
\text { Tirman, Nekmaria, Bou Sfer, Nis- } \\
\text { serghin, Bossuct, Djorf Torba, } \\
\quad \text { Ziar } \ldots \ldots \ldots \ldots \ldots \ldots \ldots \ldots \ldots\end{array}$ & 15 & 5 & 1 & \\
\hline 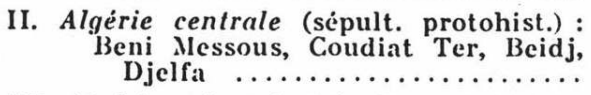 & 7 & 10 & 2 & \\
\hline $\begin{array}{l}\text { III. Algérie orientale (sépult. protohist.) : } \\
\text { Roknia, Bou Mlerzoug, Bou Nouara, } \\
\text { Sigus, Tiddis, Gastel, Dj. Osmor, } \\
\text { Mistiri, Sila, Ain el Bey, Medra- } \\
\text { cen (1) ....................... }\end{array}$ & 81 & $\underline{63}$ & $\underline{11}$ & $\underline{3}$ \\
\hline Total Algérie $\ldots \ldots \ldots \ldots \ldots \ldots \ldots \ldots$ & $\overline{103}$ & $\overline{78}$ & $\overline{14}$ & $\overline{3}$ \\
\hline $\begin{array}{l}\text { IV. Tunisie (sépult. protohist.) } \\
\text { Teboursouk, Ain Hedja, Ellez, } \\
\text { Dougga, Iraktar } \ldots \ldots \ldots \ldots \ldots \ldots \ldots\end{array}$ & 2 & 5 & 1 & \\
\hline $\begin{array}{l}\text { Sépult. puniques : } \\
\text { Carthage, Nahdiah } \ldots \ldots \ldots \ldots \ldots\end{array}$ & $\underline{20}$ & $\underline{13}$ & 2 & \\
\hline Total Tunisie $\ldots \ldots \ldots \ldots \ldots \ldots \ldots \ldots$ & 22 & $\underline{18}$ & 3 & _ \\
\hline Total Algérie et Tunisic $\ldots \ldots \ldots \ldots \ldots \ldots$ & 125 & 96 & 17 & 3 \\
\hline
\end{tabular}

(1) Nous n'avons pu retrouver trace des crànes du Medracen. Mesures de Bertholon et Chantre, 1913, Letourneau et Papillault, 1896 et de Bouchereau (inédites).

Distribution de la capacité crânienne chez les Protohistoriques d'Algérie orientale (trait plein) et chez les Puniques de Tunisie (tirets).
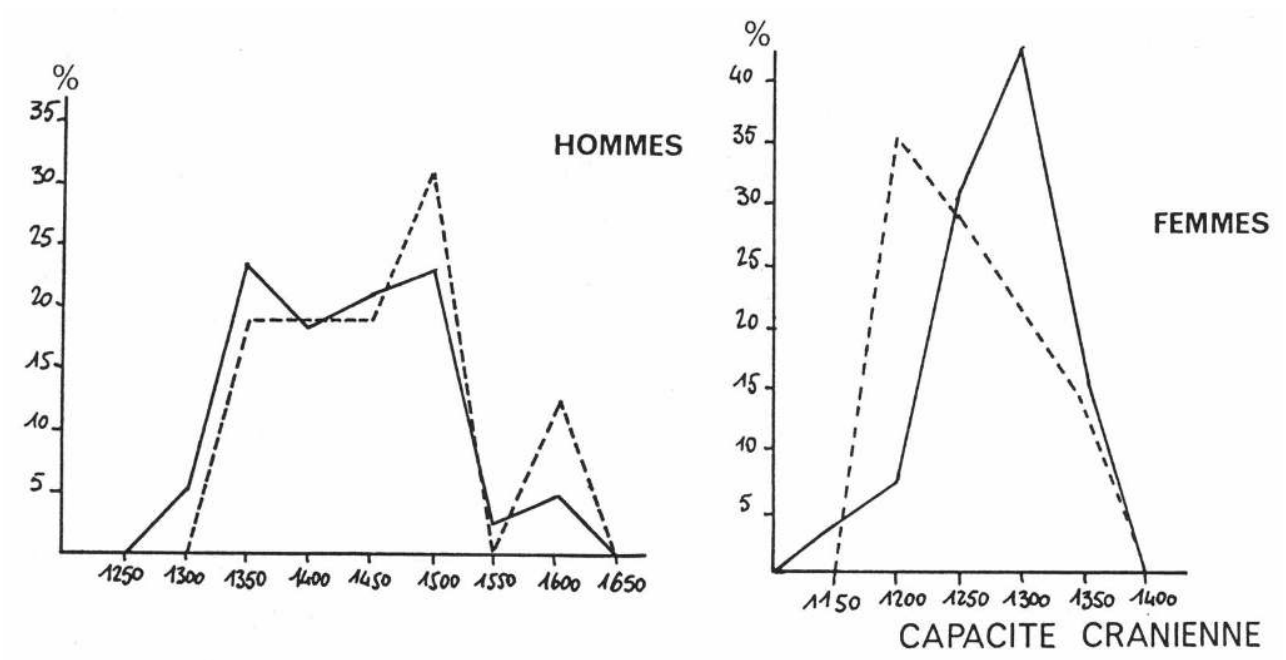

A ces trois séries nous avons joint, à titre comparatif, une quatrième constituée par des squelettes trouvés dans les sépultures romaines de l'est et du centre de l'Algérie qui ont été étudiées par Chabeuf et Boulinier en 1971.

La détermination de l'âge à la mort a pu être faite d'après l'état de synostose des sutures. Trois catégories ont été retenues pour les adultes. Il n'existe aucun crâne montrant des signes de sénescence avancée. Dans la catégorie adultes jeunes (20-30 ans) ont été rangés les crânes dont aucune suture n'était fermée, sauf la suture du basisphénoïde. Une seconde catégorie adultes moyens (30-50 ans) groupe les crânes dont la suture coronale ou sagittale, ou les deux, était en cours de fermeture. Dans la troisième catégorie, les adultes âgés de plus de 50 ans, toutes les sutures étaient fermées (ou en cours de fermeture pour la lambdoïde). Le tableau II indique la répartition des sujets par 
âge, sexe et région. La majorité des sujets se groupe dans la catégorie adultes moyens, sauf les hommes puniques dont l'âge à la mort parait avoir été sensiblement plus tardif que chez les hommes protohistoriques d'Algérie. Un pourcentage un peu plus élevé de femmes que d'hommes sont mortes plus jeunes, mais la différence n'est pas suffisamment accusée dans le nombre absolu des sujets pour qu'elle mérite d'être retenue avec certitude. L'âge à la mort de 8 enfants a été déterminé d'après le stade d'éruption dentaire. Cet âge s'échelonne entre 3 et 15 ans, la moitié des sujets étant âgés de 6 ans.

Tableau II. - Répartition des adultes par âge.

\begin{tabular}{|c|c|c|c|c|c|c|c|}
\hline & \multicolumn{2}{|c|}{$\begin{array}{c}\text { Adultes jeunes } \\
20-30 \text { ans }\end{array}$} & \multicolumn{2}{|c|}{$\begin{array}{l}\text { Adultes moyens } \\
30-50 \text { ans }\end{array}$} & \multicolumn{2}{|c|}{$\begin{array}{l}\text { Adultes âgés } \\
\text { Plus de } 50 \text { ans }\end{array}$} & \multirow{2}{*}{$\frac{\text { Total }}{\mathrm{x}}$} \\
\hline & $N$ & $\%$ & $\mathbf{N}$ & $\%$ & N & $\%$ & \\
\hline \multicolumn{8}{|l|}{ lgérie: } \\
\hline$\underset{\text { Femmes } \ldots \ldots \ldots \ldots}{\text { Homme }}$ & $\begin{array}{l}18 \\
17\end{array}$ & $\begin{array}{l}27,6 \\
34\end{array}$ & $\begin{array}{l}29 \\
23\end{array}$ & $\begin{array}{l}44,6 \\
46\end{array}$ & $\begin{array}{l}18 \\
10\end{array}$ & $\begin{array}{l}27,6 \\
20\end{array}$ & $\begin{array}{l}65 \\
50\end{array}$ \\
\hline \multicolumn{8}{|l|}{ unisie : } \\
\hline $\begin{array}{r}\text { Hommes } \ldots \ldots \ldots \\
\text { Femmes } \ldots \ldots \ldots\end{array}$ & $\begin{array}{l}4 \\
5\end{array}$ & $\begin{array}{l}21 \\
29,4\end{array}$ & $\begin{array}{l}5 \\
7\end{array}$ & $\begin{array}{l}26,3 \\
41,1\end{array}$ & $\begin{array}{r}10 \\
5\end{array}$ & $\begin{array}{l}52,6 \\
29,4\end{array}$ & $\begin{array}{l}19 \\
17\end{array}$ \\
\hline
\end{tabular}

\section{Caractères descriptifs des crânes} accentuée. Les femmes sont sensiblement plus graciles. Les parois crâniennes ont une épaisseur moyenne : $6 \mathrm{~mm}$ en moyenne aux bosses frontales, qui augmente d'avant en arrière, $6,7 \mathrm{~mm}$ au niveau du pariétal, min. $4 \mathrm{~mm}$, max. $12 \mathrm{~mm}$ (Beni Messous) et 13 (Djebel Mistiri). L'épaisseur semble plus faible chez les Puniques (moyenne 5,6 $\mathrm{mm}$ au frontal, $6 \mathrm{~mm}$ au pariétal) que chez les Protohistoriques d'Algérie. Les parois sont moins épaisses chez les femmes ( 0,1 à $0,5 \mathrm{~mm}$ en moins). Les reliefs occipitaux sont en général moyennement accentués. Chez certains individus, ils sont tout à fait effacés, alors que chez d'autres ils apparaissent assez vigoureux. Chez les femmes, en règle générale, ils sont plutôt effacés. La saillie de l'inion est variable, effacée à très accusée. Les apophyses mastoïdes apparaissent souvent assez développées chez les hommes et parfois très vigoureuses, alors que chez la majorité des femmes elles sont réduites. Le dimorphisme sexuel apparaît ainsi assez prononcé.

de la mastoïde, on note l'existence d'une crête sus-mastoïdienne sur beaucoup de crânes masculins et féminins, mais elle est rarement accentuée. En vue faciale et latérale, les arcades sus-orbitaires ont un développement variable et réparti de façon analogue dans les trois catégories que nous avons retenues (faible, moyenne, forte) chez les hommes algériens. Sur les crânes puniques, on note des variations similaires. Sur les crânes féminins des deux séries, les arcades sont le plus souvent effacées. La glabelle présente un développement moyen (catégorie $\mathrm{n}^{\circ} 3$ de Broca).

En vue supérieure, la forme du crâne est généralement ovoïde, certains individus ont une voûte pentagonoïde avec des bosses pariétales accusées, tandis que d'autres présentent une forme nettement allongée, ellipsoïde. Les sutures crâniennes sont dans l'ensemble sinueuses chez les deux sexes. En vue latérale, l'occipital forme une saillie variable, modérée à accentuée chez les Algériens, plus souvent marquée chez les Puniques. On note l'existence d'un chignon occipital à l'état d'ébauche ou franchement 
différencié sur près de $30 \%$ des crânes masculins d'Algérie, alors que chez les femmes cette caractéristique est beaucoup moins fréquente (16\%). A l'inverse, elle est beaucoup plus répandue chez les femmes puniques.

21 Le nombre des orbites basses est beaucoup moins élevé en utilisant la classification proposée pour l'indice : $18 \%$ des hommes et des femmes protohistoriques et $33 \%$ des Puniques. Hommes et femmes se classent principalement dans la catégorie moyenne de l'indice.

22 Le nez a une largeur moyenne en valeur absolue, sa hauteur est assez élevée. La répartition individuelle de l'indice est cependant assez variable avec un nombre analogue d'individus lepto- et mésorhiniens chez les hommes protohistoriques, puniques et romains. Les femmes sont davantage mésorhiniennes. On note en outre l'existence d'une proportion non négligeable d'individus platyrhiniens ( $25 \%$ des hommes et des femmes) dans les sépultures protohistoriques et romaines d'Algérie. Ils sont beaucoup plus rares dans les sépultures puniques.

23 L'angle du prognathisme a été mesuré directement dans la région alvéolaire. La variabilité est grande parmi les hommes protohistoriques algériens qui se répartissent également entre les catégories prognathe, mésognathe et orthognathe. Ces résultats sont à rapprocher de ceux fournis par l'indice nasal.

24 La taille était élevée chez les hommes (moyenne $173 \mathrm{~cm}$ ), plus basse semble-t-il chez les puniques (moyenne $167 \mathrm{~cm}$ ). Le dimorphisme sexuel est important, les femmes protohistoriques se répartissant dans les catégories sur-moyenne et élevée (moyenne $159 \mathrm{~cm}$ ), alors que les hommes se groupent pour la plupart dans la catégorie élevée.

Tableau III- Mesures des crânes masculins des sépultures protohistoriques, puniques et romaines d'Afrique du Nord.

Tableat II - Mesures des cränes masculins des.sépultures protohistoriques, puniques el romaines d'Afrique du Nord.

\begin{tabular}{|c|c|c|c|c|c|c|c|c|c|c|c|c|c|c|c|c|c|c|}
\hline & \multicolumn{6}{|c|}{ Sépultures protohistoriques } & \multirow{2}{*}{\multicolumn{3}{|c|}{ Tot. Algérie }} & \multirow{2}{*}{\multicolumn{3}{|c|}{$\begin{array}{c}\begin{array}{c}\text { Sép. protohist. } \\
\text { et puniques }\end{array} \\
\text { Tunisie }\end{array}$}} & \multirow{2}{*}{\multicolumn{3}{|c|}{$\begin{array}{c}\begin{array}{c}\text { Protohist. } \\
+ \text { Puniques }\end{array} \\
\begin{array}{c}\text { Tot. Alg. } \\
\text { - + Tunisie }\end{array}\end{array}$}} & \multirow{2}{*}{\multicolumn{3}{|c|}{\begin{tabular}{|c|} 
Scp. romaines (2) \\
Centre et \\
- Est Alg. \\
\end{tabular}}} \\
\hline & \multicolumn{3}{|c|}{$\begin{array}{c}\text { Ouest }+ \\
\text { Centre Alg. }\end{array}$} & \multicolumn{3}{|c|}{ Est Algérie } & & & & & & & & & & & & \\
\hline & $\mathrm{N}$ & M & $\sigma$ & $x$ & II & $\sigma$ & $x$ & $M$ & $\sigma$ & $\mathrm{x}$ & M & $\sigma$ & $x$ & M & $\sigma$ & $\mathrm{N}$ & M & $\sigma$ \\
\hline Longu & 13 & 191 & 7,74 & +7 & & & 60 & 188,67 & 6,57 & 177 & & 02 & 77 & 188,16 & 6,12 & 23 & 191,94 & \\
\hline & $\int_{13}^{1}$ & & 4.77 & 48 & & 4.95 & 61 & 138,69 & 4,89 & 18 & & 7,35 & 79 & & 5.49 & 21 & $1+1,00$ & \\
\hline . & 13 & 114,76 & 3,90 & 48 & 116.38 & 5,34 & 61 & 116,02 & 5,07 & 18 & 115,45 & 5,52 & 79 & 115,90 & 5,16 & 17 & 115,89 & 6, \\
\hline aut & $1 \frac{12}{0}$ & $\begin{array}{r}98,95 \\
3585\end{array}$ & 3,51 & 48 & & $\begin{array}{r}4.86 \\
-8 .\end{array}$ & 60 & $96,4 ?$ & 4.74 & 17 & 98,68 & 4,62 & 77 & 97,00 & 4,77 & 22 & 100,67 & \\
\hline nut & ${ }_{10}^{9}$ & $\begin{array}{l}135,88 \\
113\end{array}$ & $=8$. & 30 & $\begin{array}{l}136.85 \\
11389\end{array}$ & 5,85 & 39 & 136,61 & 5.70 & 15 & 134.63 & 5,82 & 54 & 136,04 & 5,76 & 18 & 135,26 & 4. \\
\hline au & . & $\begin{array}{l}113,45 \\
380,11\end{array}$ & [., 82 & 34 & $\begin{array}{r}113,87 \\
377,35\end{array}$ & $\begin{array}{r}4,98 \\
1,15\end{array}$ & 44 & $\begin{array}{l}113.78 \\
337\end{array}$ & 5,10 & 16 & $\begin{array}{l}113,09 \\
37,60\end{array}$ & $\begin{array}{r}5,46 \\
1,\end{array}$ & 60 & 113.60 & 5,16 & 9 & 114,00 & - \\
\hline & 13 & $\begin{array}{l}380,211 \\
129,21\end{array}$ & $\overline{4}, 56$ & $\begin{array}{l}36 \\
49\end{array}$ & $\begin{array}{l}377.35 \\
128.79\end{array}$ & $\begin{array}{r}12,15 \\
6 .+8\end{array}$ & $\begin{array}{l}45 \\
62\end{array}$ & $\begin{array}{l}377.75 \\
128.88\end{array}$ & $\begin{array}{r}14,86 \\
6,27\end{array}$ & 16 & & $\begin{array}{l}11,50 \\
657\end{array}$ & 61 & $\begin{array}{l}377,35 \\
128,01\end{array}$ & $\begin{array}{r}14,10 \\
6,30\end{array}$ & - & - & - \\
\hline & $l_{12}^{10}$ & 113 & ., 304 & +7 & & $\begin{array}{r}6 .+8 \\
+0.96\end{array}$ & 62 & $\begin{array}{l}128,88 \\
113.25\end{array}$ & (6,27 & 18 & & 6,97 & 80 & & 6.30 & & $=$ & 二 \\
\hline & 14 & 130,95 & 11,61 & $\begin{array}{l}+1 \\
49\end{array}$ & 129 & $7,+1$ & 63 & $\begin{array}{l}129,72 \\
129,\end{array}$ & $\begin{array}{l}7,+12 \\
8,43\end{array}$ & $\begin{array}{l}18 \\
18\end{array}$ & & $\begin{array}{l}9,90 \\
4,95\end{array}$ & $\begin{array}{l}11 \\
81\end{array}$ & & 7.05 & 二 & $\overline{-}$ & 二 \\
\hline & 10 & & 9,06 & 39 & & 9,15 & ti! & & 0,03 & 17 & & 6,78 & 66 & & 8,46 & & & \\
\hline & 10 & 527,45 & 13,50 & +2 & 522,60 & 15,00 & 52 & 523,50 & 14.75 & 15 & 527 & 12,65 & 67 & 524,21 & 14.49 & 8 & 521,25 & - \\
\hline 1 & 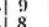 & $\begin{array}{c}1(12), 44 \\
0.34\end{array}$ & - & 28 & 1104 & 5,54 & 37 & & & 14 & & $\begin{array}{l}4,38 \\
, 3 .\end{array}$ & 51 & 104,17 & 5,14 & & & \\
\hline n & ${ }_{11}^{8}$ & $\begin{array}{l}93 \\
33 \\
33\end{array}$ & & 24 & & $\begin{array}{l}7.44 \\
3.44\end{array}$ & 32 & $\begin{array}{l}97.25 \\
3315\end{array}$ & $\begin{array}{l}6,74 \\
364\end{array}$ & 12 & 97,11 & & 44 & 97,21 & 6,24 & & - & \\
\hline 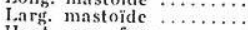 & 11 & $\begin{array}{l}3.3,13 \\
21,67\end{array}$ & $\begin{array}{l}., 1,50 \\
3,50\end{array}$ & $\begin{array}{l}45 \\
+5\end{array}$ & $\begin{array}{l}33,17 \\
32127\end{array}$ & $\begin{array}{l}3,44 \\
+, 12\end{array}$ & \begin{tabular}{|l}
56 \\
56
\end{tabular} & $\begin{array}{l}33,15 \\
2.29\end{array}$ & $\begin{array}{r}3,64 \\
+, 08\end{array}$ & $\begin{array}{l}18 \\
18\end{array}$ & $\begin{array}{l}35,17 \\
2,07\end{array}$ & $\begin{array}{r}4,48 \\
+, 24\end{array}$ & $\begin{array}{l}74 \\
74\end{array}$ & $\begin{array}{l}3,3,67 \\
21195\end{array}$ & $\begin{array}{l}3,94 \\
4,26\end{array}$ & $=$ & 二 & 二 \\
\hline & 9 & & - & & 70,75 & 4.32 & 40 & 71.19 & 4,46 & 15 & & & 55 & 71,45 & 4,70 & 18 & 71,83 & \\
\hline & 8 & 133, & - & 30 & 131 & 7,53 & 38 & 131 & & 15 & 13. & 2 & 53 & 132 & 6,72 & 15 & 134,44 & 7, \\
\hline & & $19,8 \overline{5}$ & - & 29 & & & 36 & & & 14 & & & & & & & & \\
\hline tín & 8 & 41 & - & 36 & +2 & 2,15 & 44 & $+2,07$ & 2,11 & & & & & 42 & 2,10 & 12 & $\begin{array}{r}43,03 \\
42=0\end{array}$ & 1 . \\
\hline & 9 & 32,88 & 二 & 335 & 33 & 1,74 & $4+4$ & 33.55 & & 15 & 34,05 & & & & 2,13 & 15 & $\begin{array}{r}33,58 \\
-5,53\end{array}$ & 2, \\
\hline & $\begin{array}{l}8 \\
8\end{array}$ & $\begin{array}{l}51,50 \\
24,87\end{array}$ & - & 32 & 51.31 & 2,84 & $\begin{array}{l}40 \\
41\end{array}$ & 51,45 & $\begin{array}{l}2,66 \\
0,00\end{array}$ & 15 & 52,15 & & & 21,63 & 188 & & $\begin{array}{l}52,53 \\
26,45\end{array}$ & 3, \\
\hline & $i_{i}$ & $\begin{array}{r}-7,81 \\
6,83\end{array}$ & $=$ & 23 & 8. & 1,34 & 29 & $\begin{array}{r}2.0,3.3 \\
7,83\end{array}$ & 1,35 & 11 & $\begin{array}{r}25,12 \\
8,09\end{array}$ & $\begin{array}{l}1,40 \\
0,69\end{array}$ & 40 & $\begin{array}{r}2.4 .41 \\
7,90\end{array}$ & $\begin{array}{l}1,88 \\
1,18\end{array}$ & 18 & 26,45 & $\underline{-}^{2,66}$ \\
\hline & 6 & & & 24 & 79 & 7,74 & 30 & 80.95 & & 12 & 80, & 3,96 & i2 & 80,95 & 6.63 & & & \\
\hline & & 1447,45 & 101,00 & 38 & 1456,95 & 75,50 & 4!) & 54,95 & 80,50 & & 480,9 & 79,00 & & 461.45 & 80,50 & 21 & 565, & \\
\hline
\end{tabular}

(1) Moyennes statistiques et écarts-types calculés it partir de 10 sujets. Au-dessous de 10 sujets, les moyennes sont arithmétiques. (2) 11 et $\sigma$, calculs personnels complëtes par des mesures personnelles. 
Tableau IV- Mesures des crânes féminins des sépultures protohistoriques, puniques et romaines.

\begin{tabular}{|c|c|c|c|c|c|c|c|c|c|c|c|c|c|c|c|c|c|c|}
\hline & \multicolumn{9}{|c|}{ Sépultures protohistoriques } & \multirow{2}{*}{\multicolumn{3}{|c|}{$\begin{array}{c}\text { Sép. protohist. } \\
\text { et puniques }\end{array}$}} & \multirow{2}{*}{\multicolumn{3}{|c|}{$\begin{array}{l}\begin{array}{c}\text { Protohist. } \\
+ \text { Puniques }\end{array} \\
\begin{array}{l}\text { Tot. Alg. } \\
\text { + Tunisie }\end{array} \\
\end{array}$}} & \multirow{2}{*}{\multicolumn{3}{|c|}{$\begin{array}{l}\text { Sép. romaines } \\
\text { Centre et } \\
\text { Est Alg. } \\
\end{array}$}} \\
\hline & \multicolumn{3}{|c|}{$\begin{array}{c}\text { Ouest +t } \\
\text { Centre Aig. }\end{array}$} & \multicolumn{3}{|c|}{ Est Algérie } & \multicolumn{3}{|c|}{ Tot. Alg. } & & & & & & & & & \\
\hline & $\mathrm{N}$ & M & $\sigma$ & $\mathrm{N}$ & M & & $\mathrm{N}$ & M & $\sigma$ & N & M & $\sigma$ & $\mathrm{N}$ & M & $\sigma$ & $\mathrm{s}$ & $\mathrm{M}$ & \\
\hline Longueur max. & 7 & 179,85 & - & & 180,69 & & & & & & 30 & & & 79,67 & & 16 & 179,70 & 4,83 \\
\hline Largeu & 7 & $\begin{array}{l}131,42 \\
131,0\end{array}$ & $=$ & 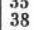 & $\begin{array}{l}\begin{array}{l}180,69 \\
136,50\end{array} \\
\end{array}$ & $\begin{array}{l}4,98 \\
4,86\end{array}$ & 45 & $\begin{array}{l}180,66 \\
135,63\end{array}$ & $\begin{array}{l}4,80 \\
5,19\end{array}$ & 17 & 2 & $\begin{array}{l}5,53 \\
5,88\end{array}$ & 62 & 79,67 & & $\begin{array}{l}16 \\
16\end{array}$ & $\begin{array}{l}179,13 \\
134,13\end{array}$ & $\begin{array}{l}4,83 \\
6,21\end{array}$ \\
\hline Lar & 8 & 108,12 & - & 3 & $\begin{array}{l}114,01 \\
11400\end{array}$ & $\begin{array}{l}7,010 \\
4,05\end{array}$ & $\begin{array}{l}45 \\
42\end{array}$ & $\begin{array}{l}150,00 \\
112,93\end{array}$ & $\begin{array}{l}4,83 \\
4,83\end{array}$ & 17 & 112,78 & $\begin{array}{l}4,00 \\
4,08\end{array}$ & $\begin{array}{l}02 \\
59\end{array}$ & 112.90 & $\begin{array}{l}5,04 \\
4,59\end{array}$ & 10 & 113,35 & 0, \\
\hline$\because$ & 8 & 93,37 & - & 4 & $\begin{array}{r}17,45 \\
94,45\end{array}$ & $\begin{array}{l}4,00 \\
4,17\end{array}$ & \begin{tabular}{|l}
72 \\
12
\end{tabular} & 94,33 & 4,02 & 16 & $\begin{array}{r}94,09 \\
94,0\end{array}$ & 2,64 & 58 & 94,27 & & & 94,73 & \\
\hline$\because$ & 4 & $\overline{11}$ & - & 22 & 131,33 & 5,88 & 26 & 131,24 & & 11 & 128,18 & & 37 & 130,34 & 5,22 & 4 & 129,4 & 4, \\
\hline $\begin{array}{l}\text { Haut } \\
\text { C. sa }\end{array}$ & 5 & $\begin{array}{l}111,00 \\
36100\end{array}$ & $\bar{z}$ & 24 & 112,22 & 5,43 & 29 & 112,01 & 5,04 & 14 & 109,82 & 4,11 & 43 & 111,29 & 4,77 & 8 & 108,6 & - \\
\hline Si & 9 & $\begin{array}{l}361,00 \\
126,22\end{array}$ & $=$ & $\begin{array}{l}21 \\
36\end{array}$ & $\begin{array}{l}366,50 \\
125,37\end{array}$ & 13,45 & 26 & $\begin{array}{l}364,95 \\
125\end{array}$ & $\begin{array}{r}14,10 \\
5,82\end{array}$ & 15 & $\begin{array}{l}359,45 \\
12510\end{array}$ & 10,50 & 41 & $\begin{array}{l}363,05 \\
102,0\end{array}$ & 13,11 & - & 二 & - \\
\hline & 9 & $\begin{array}{l}120,22 \\
109,88\end{array}$ & $=$ & & $\begin{array}{l}125,37 \\
109,91\end{array}$ & $\begin{array}{l}5,91 \\
4,38\end{array}$ & $\begin{array}{l}45 \\
44\end{array}$ & $\begin{array}{l}125,73 \\
110,03\end{array}$ & $\begin{array}{r}5,82 \\
4,24\end{array}$ & 17 & & & 62 & & $\begin{array}{l}5,43 \\
3,96\end{array}$ & 二 & 二 & 二 \\
\hline & 7 & $\begin{array}{l}109,88 \\
130,28\end{array}$ & ב & 8 & $\begin{array}{l}109,91 \\
125,61\end{array}$ & $\begin{array}{l}4,38 \\
8,31\end{array}$ & 45 & $\begin{array}{l}110,03 \\
125,13\end{array}$ & $\begin{array}{l}4,24 \\
0,27\end{array}$ & $\begin{array}{l}17 \\
17\end{array}$ & 73 & $\begin{array}{l}2,98 \\
6,63\end{array}$ & $\begin{array}{l}61 \\
62\end{array}$ & $\begin{array}{l}109,67 \\
125,70\end{array}$ & $\begin{array}{l}3,96 \\
7.71\end{array}$ & 二 & 二 & 二 \\
\hline C & 6 & $\begin{array}{l}100,20 \\
108,33\end{array}$ & 二 & 26 & $\begin{array}{l}126,01 \\
114,76\end{array}$ & $\begin{array}{l}8,51 \\
7,53\end{array}$ & 32 & 113,68 & 7,71 & $\begin{array}{l}17 \\
15\end{array}$ & 112,06 & $\begin{array}{l}0,63 \\
5,76\end{array}$ & \begin{tabular}{|l}
62 \\
47
\end{tabular} & $\begin{array}{l}12.0,10 \\
113,17\end{array}$ & 7,14 & & & \\
\hline & 7 & 497,71 & 二 & 30 & $\begin{array}{l}114,61 \\
507,45\end{array}$ & $\begin{array}{r}7,2,33 \\
12,20\end{array}$ & 37 & $\begin{array}{l}113,68 \\
505,60\end{array}$ & 11,80 & 15 & $\begin{array}{l}112,06 \\
499,45\end{array}$ & $\begin{array}{r}5,76 \\
12,35\end{array}$ & 52 & 501,15 & $\begin{array}{r}7,14 \\
12,15\end{array}$ & 6 & 507,00 & - \\
\hline $\mathrm{L}$ & 3 & 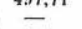 & - & $\begin{array}{l}30 \\
19\end{array}$ & $\begin{array}{l}99,81 \\
99,8)\end{array}$ & $\begin{array}{r}12,20 \\
+, 40\end{array}$ & 22 & $\begin{array}{r}9,0,00 \\
993\end{array}$ & $\begin{array}{r}1,80 \\
4,40\end{array}$ & ${ }_{11}^{12}$ & $\begin{array}{r}49,9,43 \\
95,85\end{array}$ & $\begin{array}{r}1,350 \\
4,14\end{array}$ & 33 & 98.15 & $\begin{array}{r}12,15 \\
4,48\end{array}$ & & & \\
\hline 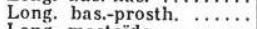 & 2 & $=$ & $=$ & 10 & 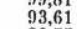 & $\begin{array}{l}7,+70 \\
3,56\end{array}$ & 14 & 93,51 & & $\begin{array}{r}11 \\
9\end{array}$ & . & - & 23 & 93,11 & $\begin{array}{l}7,+60 \\
3,00\end{array}$ & & & \\
\hline $\bar{L}$ & 6 & 29,50 & 二 & 3 & & & 38 & & & 16 & 29,07 & 3,24 & 54 & 28,96 & $\begin{array}{l}3,12 \\
3,12\end{array}$ & - & - & - \\
\hline $\mathrm{L}$ & 6 & 16,33 & - & 10 & 17,31 & 2,36 & 38 & 17,25 & 2,40 & 16 & & 2,76 & 54 & 17,13 & 2,50 & 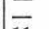 & $\overline{-}$ & $\overline{-}$ \\
\hline Hau & 4 & - & - & 17 & 68,73 & 4,52 & 21 & & 4, & 10 & & 4,04 & 31 & 67,33 & 4,38 & 11 & 70.95 & 4,00 \\
\hline Dia & 3 & - & - & 16 & 123,31 & 5,88 & 19 & 122,08 & & 10 & 122,65 & 4,29 & 29 & 122,26 & 5,64 & 11 & 124,63 & 2,23 \\
\hline Lar & 4 & - & - & is & 20 & 1,82 & 20 & & & 10 & 20,15 & 2,36 & 30 & 20,27 & & - & & - \\
\hline lit & 4 & - & - & 18 & 40,95 & 1,50 & 22 & 41,17 & & 10 & 40,65 & 2,44 & 32 & 41,01 & 1,78 & 9 & 41,44 & \\
\hline te $\ldots$. & 5 & 32,20 & - & 19 & 33,66 & 2,30 & 24 & 33,49 & 2,05 & 10 & 32,75 & 1,42 & 34 & 33,25 & 1,93 & 11 & 33,81 & 1,21 \\
\hline $\mathrm{Ha}$ & 4 & 二 & - & 18 & 49,41 & 3,06 & 22 & 48,51 & 3,48 & 10 & 47,15 & 2,54 & 32 & 48,03 & 3,14 & 12 & 51,95 & 3,02 \\
\hline lat & 4 & $\bar{z}$ & 二 & 8 & 24,61 & 0,98 & 22 & 24,49 & 0,94 & 10 & 23,65 & 1,83 & 32 & 24,17 & 1,46 & 13 & 25,52 & 2,43 \\
\hline & $\begin{array}{l}3 \\
2\end{array}$ & $\overline{-}$ & 二 & 15 & $\begin{array}{r}61,59 \\
81,55\end{array}$ & $\begin{array}{l}0,80 \\
6,03\end{array}$ & $\begin{array}{l}18 \\
15\end{array}$ & $\begin{array}{r}6,40 \\
80,05\end{array}$ & $\begin{array}{l}0,93 \\
8,07\end{array}$ & 10 & $\begin{array}{r}6,95 \\
76,42\end{array}$ & & ${ }_{22}^{28}$ & $\begin{array}{r}6,599 \\
79,03\end{array}$ & $\begin{array}{l}0,93 \\
6,84\end{array}$ & - & - & 二 \\
\hline Capacité crân. & & 1302,40 & - & 26 & 303,45 & 50,00 & 31 & 1303,95 & 45,50 & & 281,95 & $\overline{55}, 00$ & 451 & 1296,954 & 48,50 & $1+1$ & 1295,90 & $\overline{43}, 0 \mathrm{r}$ \\
\hline
\end{tabular}

Tableau V - Indices des crânes masculins et féminins des sépultures protohistoriques, puniques et romaines.

\begin{tabular}{|c|c|c|c|c|c|c|c|c|}
\hline \multicolumn{9}{|c|}{ I. - Hommes. } \\
\hline & \multicolumn{4}{|c|}{ Sépultures protohistoriques } & \multirow{2}{*}{ Tot. Algérie } & \multirow{2}{*}{$\begin{array}{c}\begin{array}{c}\text { Sép. protohlst. } \\
\text { et puniques }\end{array} \\
\text { Tunisie } \\
\end{array}$} & \multirow{2}{*}{$\begin{array}{c}\text { Protohist. } \\
\text { + Puniques } \\
\text { Tot. Alg. } \\
\text { + Tunisie } \\
\end{array}$} & \multirow{2}{*}{\begin{tabular}{|c|} 
Sép. romaines \\
Centre + Est Alg. \\
\end{tabular}} \\
\hline & \multicolumn{2}{|c|}{$\begin{array}{c}\begin{array}{c}\text { Ouest }+ \\
\text { Centre AIg. }\end{array} \\
\text {. }\end{array}$} & \multicolumn{2}{|c|}{ Est Algérie } & & & & \\
\hline $\begin{array}{l}\text { Ind. crânien } \\
\text { Indd. haut.-long. (por.) }\end{array}$ & \begin{tabular}{|rrr} 
N & M. \\
13 & 72,65 & 4 \\
10 & 61,15 & 2 \\
10 & 83,15 & 5 \\
9 & 83,91 & 5 \\
12 & 85,99 & 2 \\
12 & 72,27 & 3 \\
13 & 88,22 & 2 \\
8 & 54,26 & 2 \\
8 & 56,06 & - \\
7 & 74,60 & - \\
8 & 79,31 & $=$ \\
8 & 48,30 & $=$
\end{tabular} & $\begin{array}{l}\sigma \\
4,68 \\
2,62 \\
5,56 \\
2,90 \\
3,96 \\
2,48 \\
= \\
= \\
=\end{array}$ & \begin{tabular}{|ccc}
$\mathrm{N}$ & $\mathrm{M}$ \\
44 & 73,77 & 3 \\
34 & 60,49 & 2 \\
34 & 82,61 & 4 \\
29 & 83,63 & 3 \\
45 & 82,73 & 3 \\
43 & 68,69 & 3 \\
47 & 87,70 & 1 \\
28 & 53,71 & 1 \\
30 & 94,83 & 4 \\
29 & 72,53 & 4 \\
35 & 79,30 & 5 \\
32 & 50,39 &
\end{tabular} & \begin{tabular}{c|}
$\sigma$ \\
$\sigma$, \\
3,10 \\
2,28 \\
4,06 \\
3,94 \\
3,36 \\
3,52 \\
1,34 \\
4,04 \\
4,44 \\
4,22 \\
5,01 \\
4,84
\end{tabular} & 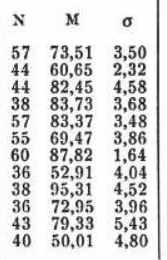 & $\begin{array}{ccc}\mathrm{N} & \mathrm{M} & \sigma \\
17 & 74,25 & 4,34 \\
15 & 59,61 & 3,26 \\
16 & 80,33 & 4,04 \\
15 & 81,61 & 3,82 \\
17 & 84.61 & 4,18 \\
17 & 70,37 & 3,72 \\
18 & 88,23 & 0,94 \\
15 & 53,65 & 3,64 \\
15 & 95,75 & 5,74 \\
15 & 73,47 & 4,02 \\
15 & 79,06 & 6,09 \\
15 & 48,43 & 4,92\end{array}$ & 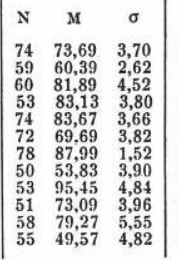 & \begin{tabular}{|ccc}
$\mathrm{N}$ & $\mathrm{M}$ & $\sigma$ \\
21 & 73,89 & $\mathbf{5}, 50$ \\
9 & 59,43 & - \\
9 & 82,38 & $\overline{1}$ \\
17 & 81,31 & 3,56 \\
17 & 85,77 & 5,24 \\
19 & 71,47 & 5,20 \\
15 & 53,21 & 4,88 \\
14 & 95,95 & 3,48 \\
15 & 74,95 & 4,26 \\
12 & 77,61 & 6,84 \\
18 & 50,63 & 7,26
\end{tabular} \\
\hline & & & & II. - & $F$ & & & \\
\hline 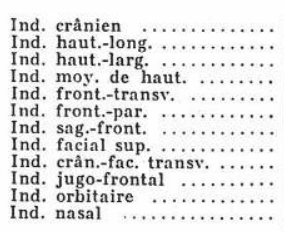 & $\mid \begin{array}{rrr}7 & 73,07 \\
5 & 61,80 \\
5 & 83,68 \\
4 & 83,6 \\
8 & 86,46 \\
6 & 70,93 \\
9 & 70,7 \\
3 & 87,07 \\
2 & = \\
3 & = \\
4 & = \\
4 & = & \end{array}$ & $\begin{array}{l}\text { 三 } \\
\equiv \\
\equiv \\
\equiv \\
\text { 三 }\end{array}$ & 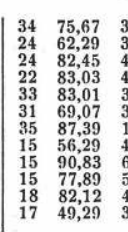 & $\begin{array}{l}3,78 \\
3,48 \\
4,92 \\
4,28 \\
3,28 \\
3,96 \\
1,76 \\
4,12 \\
6,46 \\
5,34 \\
4,44 \\
3,94\end{array}$ & 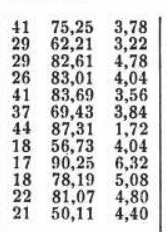 & 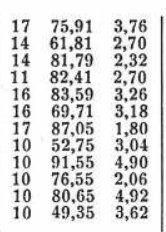 & 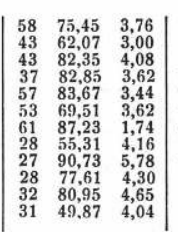 & 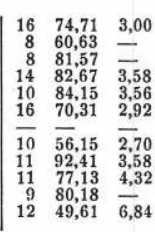 \\
\hline
\end{tabular}


TABLEAU VI - Dimensions et indices des mandibules masculines et féminines.

\begin{tabular}{|c|c|c|c|c|c|c|c|c|c|c|c|c|c|c|c|c|c|c|}
\hline & \multicolumn{9}{|c|}{ Sépultures protohistoriques } & \multirow{2}{*}{\multicolumn{3}{|c|}{$\begin{array}{c}\begin{array}{c}\text { Protoh. et } \\
\text { Puniques }\end{array} \\
\text { Tunisie }\end{array}$}} & \multirow{2}{*}{\multicolumn{3}{|c|}{$\begin{array}{c}\begin{array}{c}\text { Protoh. et } \\
\text { Puniques }\end{array} \\
\text { Tot. Alg. } \\
+ \text { Tunisie } \\
\end{array}$}} & \multirow{2}{*}{\multicolumn{3}{|c|}{$\begin{array}{c}\text { Sép. romaines } \\
\text { Centre + Est } \mathrm{Al}_{\mathrm{g}} \text {. }\end{array}$}} \\
\hline & \multicolumn{3}{|c|}{$\begin{array}{c}\text { Ouest + } \\
\text { Centre Alg. }\end{array}$} & \multicolumn{3}{|c|}{ Est Algérie } & \multicolumn{3}{|c|}{ Tot. Algéric } & & & & & & & & & \\
\hline & $\mathrm{x}$ & M- & $\sigma$ & $\mathrm{N}$ & M & $\sigma$ & $\mathrm{N}$ & M & $\sigma$ & $\mathrm{N}$ & M & $\sigma$ & $\mathrm{x}$ & M & $\sigma$ & $x$ & M & $\sigma$ \\
\hline \multicolumn{19}{|c|}{ 1. - Hommes. } \\
\hline Longue & $\begin{array}{l}5 \\
4\end{array}$ & 101,80 & 二 & $\begin{array}{r}11 \\
7\end{array}$ & $\begin{array}{l}104,45 \\
122,00\end{array}$ & $\underline{8,91}$ & 16 & $\begin{array}{l}103,62 \\
{ }_{1}^{2} 1\end{array}$ & 7,57 & 8 & 101,12 & - & & $\begin{array}{l}102,79 \\
\end{array}$ & 7,22 & 14 & & 9,68 \\
\hline Lar & 7 & $\overline{96,14}$ & $\bar{z}$ & 14 & $\begin{array}{l}122,00 \\
103,64\end{array}$ & $\overline{8,51}$ & $\begin{array}{l}11 \\
21\end{array}$ & $\begin{array}{l}121,27 \\
101,14\end{array}$ & $\begin{array}{l}7,26 \\
8,05\end{array}$ & $\begin{array}{l}6 \\
6\end{array}$ & $\begin{array}{l}127,00 \\
106,16\end{array}$ & $\bar{z}$ & $\begin{array}{l}17 \\
27\end{array}$ & $\begin{array}{l}123,29 \\
102,25\end{array}$ & $\begin{array}{l}7,20 \\
8,08\end{array}$ & \begin{tabular}{|l}
9 \\
11
\end{tabular} & $\begin{array}{l}125,66 \\
103,72\end{array}$ & 7,26 \\
\hline & 12 & 31,00 & 3,19 & 29 & 31,89 & 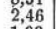 & 41 & $\begin{array}{r}31,63 \\
31,63\end{array}$ & $\begin{array}{l}2,69 \\
2,69\end{array}$ & 8 & $\begin{array}{r}30,70 \\
33,75\end{array}$ & $\overline{-}$ & 49 & 31,97 & $\begin{array}{l}8,010 \\
2,89 \\
2\end{array}$ & 6 & $\begin{array}{r}30,72 \\
31,66\end{array}$ & $\frac{1,20}{-20}$ \\
\hline & 16 & 30,87 & 2,94 & 33 & 31,36 & 1,99 & 49 & 31,20 & 2,32 & 8 & $\begin{array}{l}31,50 \\
31\end{array}$ & - & 57 & 31,24 & 2,37 & \begin{tabular}{|l|l} 
\\
\end{tabular} & 31,89 & 5,34 \\
\hline $\begin{array}{l}\text { Haut } \\
\text { Epais }\end{array}$ & $\begin{array}{l}15 \\
16\end{array}$ & $\begin{array}{l}28,60 \\
13,68\end{array}$ & $\begin{array}{l}2,35 \\
1,85\end{array}$ & $\begin{array}{l}29 \\
33\end{array}$ & $\begin{array}{l}29,34 \\
1363\end{array}$ & 2,46 & 44 & $\begin{array}{l}29,09 \\
.365\end{array}$ & 2,42 & 9 & $\begin{array}{l}29,55 \\
1500\end{array}$ & $=$ & 53 & $\begin{array}{l}29,16 \\
1134\end{array}$ & $\begin{array}{l}2,44 \\
1,75\end{array}$ & $\overline{13}$ & $\overline{13}, 83$ & \\
\hline & 15 & $\begin{array}{l}15,60 \\
15,60\end{array}$ & $\begin{array}{l}1,00 \\
1,54\end{array}$ & $\begin{array}{l}3.5 \\
33\end{array}$ & $\begin{array}{l}13,63 \\
15,54\end{array}$ & $\begin{array}{l}1,74 \\
1,60\end{array}$ & $\begin{array}{l}49 \\
48\end{array}$ & $\begin{array}{l}13,65 \\
15,58\end{array}$ & $\begin{array}{l}1,76 \\
1,56\end{array}$ & ${ }_{9}^{8}$ & $\begin{array}{l}15,00 \\
16,66\end{array}$ & 三 & $\begin{array}{l}31 \\
57\end{array}$ & $\begin{array}{l}15,8 * \\
15,75\end{array}$ & $\begin{array}{l}1,75 \\
1,60\end{array}$ & 13 & 13,83 & 1,76 \\
\hline & 15 & 16,60 & 1,40 & 31 & $\begin{array}{l}16,04 \\
16,74\end{array}$ & $\begin{array}{l}1,00 \\
1,73\end{array}$ & $\begin{array}{l}70 \\
46\end{array}$ & $\begin{array}{l}16,69 \\
16,69\end{array}$ & 1,61 & 9 & 18,00 & & 55 & $\begin{array}{l}16,90 \\
16\end{array}$ & & $-\overline{1}$ & & - \\
\hline & 8 & $\begin{array}{r}62,75 \\
3318\end{array}$ & 277 & 15 & 605,39 & 6,15 & 23 & 64,47 & 5,95 & 8 & $\begin{array}{l}63,62 \\
35,62\end{array}$ & - & 31 & 64,25 & & 14 & & $\begin{array}{l}7,11 \\
381\end{array}$ \\
\hline nia & $\begin{array}{r}11 \\
9\end{array}$ & $\begin{array}{r}339,18 \\
119,11\end{array}$ & 2,75 & $\begin{array}{l}26 \\
13\end{array}$ & & 3,24 & 37 & $\begin{array}{r}33,81 \\
\end{array}$ & 3,09 & 9 & & 二 & 46 & 34 & $\begin{array}{l}3,10 \\
713\end{array}$ & ${ }_{10}^{14}$ & & $\begin{array}{l}3,84 \\
9,27\end{array}$ \\
\hline $\begin{array}{l}\text { Angle } \\
\text { An }\end{array}$ & 11 & 67,18 & $\overline{4,53}$ & $\begin{array}{l}13 \\
24\end{array}$ & $\begin{array}{r}115,69 \\
70,54\end{array}$ & $\begin{array}{l}6,66 \\
4,96\end{array}$ & $\begin{array}{l}22 \\
35\end{array}$ & $\begin{array}{r}117,09 \\
69,48\end{array}$ & $\begin{array}{l}7,32 \\
5,02\end{array}$ & 7 & $\begin{array}{r}119,87 \\
70,00\end{array}$ & 二 & $\begin{array}{l}30 \\
42\end{array}$ & $\begin{array}{r}117,83 \\
69,57\end{array}$ & $\begin{array}{r}7,13 \\
4,89\end{array}$ & 10 & 123 & $\stackrel{9,27}{-}$ \\
\hline Ind. & $\begin{array}{l}3 \\
3\end{array}$ & $=$ & $=$ & 7 & 86,27 & - & 10 & $\begin{array}{l}88,40 \\
86,33\end{array}$ & $\begin{array}{l}3,102 \\
7,10\end{array}$ & 6 & 81,36 & - & 16 & 84,47 & 7,17 & 9 & 86,18 & - \\
\hline & $\begin{array}{c}3 \\
16\end{array}$ & $\overline{44}, 48$ & $\overline{5,90}$ & $\begin{array}{r}5 \\
32\end{array}$ & $\begin{array}{l}88,42 \\
43,44\end{array}$ & $\overline{5,47}$ & $\begin{array}{r}8 \\
48\end{array}$ & $\begin{array}{l}85,16 \\
43,79\end{array}$ & $\overline{5,58}$ & $\begin{array}{l}5 \\
8\end{array}$ & $\begin{array}{l}84,60 \\
47,98\end{array}$ & $=$ & $\begin{array}{l}13 \\
\frac{13}{6}\end{array}$ & $\begin{array}{l}84,94 \\
44,39\end{array}$ & $\begin{array}{l}6,50 \\
5,86\end{array}$ & \begin{tabular}{|l}
9 \\
13
\end{tabular} & $\begin{array}{l}81,35 \\
44,63\end{array}$ & $\overline{4,56}$ \\
\hline & 15 & $\begin{array}{r}54,99 \\
53,7\end{array}$ & 6,61 & 28 & & 5,81 & 43 & 54,01 & & 9 & & $\bar{Z}$ & 52 & $\begin{array}{l}4,439 \\
54,46\end{array}$ & $\begin{array}{l}0,00 \\
6,12\end{array}$ & & & \\
\hline Ind. br. mon & & 53,57 & - & 14 & 54,50 & 6,4 & 22 & 54,16 & 5,7 & 8 & 55,33 & - & 30 & 54,47 & 6,27 & 14 & 55,61 & 9,72 \\
\hline \multicolumn{19}{|c|}{ 11. - Femmes. } \\
\hline & & - & - & & 100,00 & - & 11 & 99,45 & 4,27 & & 93,20 & & & & 4,85 & & & \\
\hline & ${ }_{3}^{2}$ & - & & $\begin{array}{l}7 \\
8\end{array}$ & $\begin{array}{r}113 \\
91\end{array}$ & $=$ & 19 & $\begin{array}{r}111,77 \\
90,81\end{array}$ & & 3 & 二 & - & 12 & & & 4 & & - \\
\hline & 4 & $=$ & - & $\begin{array}{r}8 \\
17\end{array}$ & $\begin{array}{l}91,62 \\
29,64\end{array}$ & & $\begin{array}{l}11 \\
21\end{array}$ & $\begin{array}{l}90,81 \\
29,38\end{array}$ & $\begin{array}{l}5,56 \\
2,49\end{array}$ & $\frac{4}{5}$ & $\overline{29}, 40$ & $\bar{z}$ & & $\begin{array}{l}91 \\
29\end{array}$ & & ${ }_{3}^{6}$ & 87,83 & $=$ \\
\hline & 4 & $\overline{0}$ & - & 21 & $\begin{array}{l}28,42 \\
28,42\end{array}$ & 2,50 & 25 & $\begin{array}{l}29,3,48 \\
28,44\end{array}$ & $\begin{array}{l}2,38 \\
2,38\end{array}$ & & 28,80 & 二 & $\begin{array}{l}20 \\
30\end{array}$ & $\begin{array}{r}2,3,30 \\
28,49\end{array}$ & & 6 & $\overline{29}, 50$ & 二 \\
\hline & 6 & $\begin{array}{r}26,33 \\
\end{array}$ & $=$ & $\begin{array}{l}19 \\
22\end{array}$ & $\begin{array}{l}25,94 \\
11,77\end{array}$ & 2,06 & $\begin{array}{r}25 \\
27\end{array}$ & 26 & 2,03 & 5 & 26,00 & - & 30 & $\begin{array}{l}26,03 \\
\end{array}$ & & & - & - \\
\hline & $\begin{array}{l}5 \\
6\end{array}$ & $\begin{array}{l}12,40 \\
14,16\end{array}$ & 二 & $\begin{array}{l}22 \\
19\end{array}$ & $\begin{array}{l}12,77 \\
15,47\end{array}$ & 1,47 & $\begin{array}{l}27 \\
25\end{array}$ & $\begin{array}{l}12,70 \\
15,10\end{array}$ & 1,46 & & $\begin{array}{l}13,40 \\
15,80\end{array}$ & & 2 & $\begin{array}{l}12,81 \\
11,21\end{array}$ & & 7 & 11,85 & - \\
\hline & $\begin{array}{l}0 \\
5\end{array}$ & $\begin{array}{l}1,16 \\
16,00\end{array}$ & 二 & $\begin{array}{l}19 \\
19\end{array}$ & 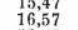 & $\begin{array}{l}1,17 \\
1,16\end{array}$ & $\begin{array}{l}25 \\
24\end{array}$ & $\begin{array}{l}15,16 \\
16,45\end{array}$ & $\begin{array}{l}1,40 \\
1,25\end{array}$ & $\begin{array}{l}5 \\
5\end{array}$ & $\begin{array}{l}15,80 \\
16,60\end{array}$ & $=$ & $\begin{array}{l}30 \\
29\end{array}$ & $\begin{array}{l}15,26 \\
16,48\end{array}$ & & 二 & $=$ & $=$ \\
\hline & 3 & $\overline{3}$ & 二 & 14 & 56,57 & & 17 & & 5,23 & 5 & 51,79 & 二 & 22 & 55,63 & & 5 & 54,80 & $=$ \\
\hline & & 31,00 & $=$ & ${ }_{11}^{14}$ & $\begin{array}{r}31,07 \\
122\end{array}$ & & 20 & $\begin{array}{r}31,04 \\
\end{array}$ & $\begin{array}{r}1,90 \\
\end{array}$ & 5 & 30,80 & -1 & 25 & $\begin{array}{r}31,00\end{array}$ & & 7 & 32,28 & - \\
\hline & $\frac{2}{4}$ & 二 & $=$ & $\begin{array}{l}11 \\
11\end{array}$ & $\begin{array}{r}122,27 \\
666,45\end{array}$ & $\begin{array}{l}6,60 \\
3,90\end{array}$ & 13 & $\begin{array}{r}121,84 \\
66,13\end{array}$ & $\begin{array}{l}6,28 \\
4,89\end{array}$ & $\begin{array}{l}5 \\
4\end{array}$ & 122,00 & - & 18 & $\begin{array}{r}121 ; 88 \\
68.52\end{array}$ & $\begin{array}{l}5,49 \\
7,06\end{array}$ & 5 & 127,00 & - \\
\hline In & ${ }_{2}^{4}$ & 二 & - & $\begin{array}{r}11 \\
7\end{array}$ & $\begin{array}{l}66,45 \\
89,11\end{array}$ & 3,90 & $\begin{array}{r}15 \\
9\end{array}$ & $\begin{array}{l}66,13 \\
89,64\end{array}$ & $\underline{4,89}$ & ${ }_{3}^{4}$ & $=$ & - & 19 & & & 4 & - & - \\
\hline & 2 & 二 & - & 6 & 80,28 & - & 8 & & - & $\begin{array}{l}3 \\
3 \\
3\end{array}$ & $\bar{Z}$ & - & ${ }_{11}^{12}$ & & & $\begin{array}{l}4 \\
4 \\
\end{array}$ & 二 & 二 \\
\hline & $\begin{array}{l}4 \\
6 \\
6\end{array}$ & $\overline{54}, 00$ & $=$ & $\begin{array}{l}21 \\
19\end{array}$ & $\begin{array}{l}45.43 \\
5908\end{array}$ & 5,77 & 25 & 45,15 & 5,47 & 5 & 46,76 & - & 30 & 45 & & 6 & 40,65 & - \\
\hline $\begin{array}{l}\text { Ind. br. mont. } \\
\text { Ind. }\end{array}$ & 3 & - & - & 15 & $\begin{array}{l}59,98 \\
54,91\end{array}$ & 6,00 & & 54,58 & $\begin{array}{l}7,32 \\
6,04\end{array}$ & 5 & $\begin{array}{l}60,98 \\
59,46\end{array}$ & & 21 & 55,74 & 5,71 & 5 & 59,84 & \\
\hline
\end{tabular}

\section{Dimorphisme sexuel}

A plusieurs reprises, nous avons souligné dans les paragraphes précédents l'existence d'un dimorphisme sexuel assez prononcé, notamment une bien moindre robustesse chez les femmes se traduisant par une gracilité générale des superstructures crâniennes contrastant avec la robustesse de beaucoup de crânes masculins; à la mandibule, absence d'extroversion de la région goniaque et effacement des reliefs osseux ; stature sensiblement moins élevée.

Ce contraste a été chiffré dans le tableau VIII où sont indiquées les différences sexuelles du crâne et de la stature. Un indice de dimorphisme sexuel a été calculé pour les Protohistoriques et les Puniques et a été comparé aux indices publiés par F. Demoulin (1972) sur des crânes d'Algériens actuels et par H. de Villiers sur des Égyptiens. On y voit qu'en ce qui concerne la série algérienne protohistorique, le dimorphisme est prononcé au niveau de la partie moyenne de la face (diamètre bizygomatique, hauteur de l'arcade zygomatique), au niveau de l'apophyse mastoïde et de la mandibule (largeurs bicondylienne et bigoniaque, hauteur de la branche horizontale, hauteur et largeur de la branche montante) et qu'il est moins accentué au niveau du neurocrâne. Chez les séries puniques, le dimorphisme apparaît encore plus prononcé. Comparativement aux Algériens actuels, les différences sexuelles sont similaires, sauf pour la hauteur de la face. Chez les Égyptiens, le dimorphisme paraît moins prononcé, sauf au niveau de la mandibule. 
Tableau VII - Dimensions des os longs.

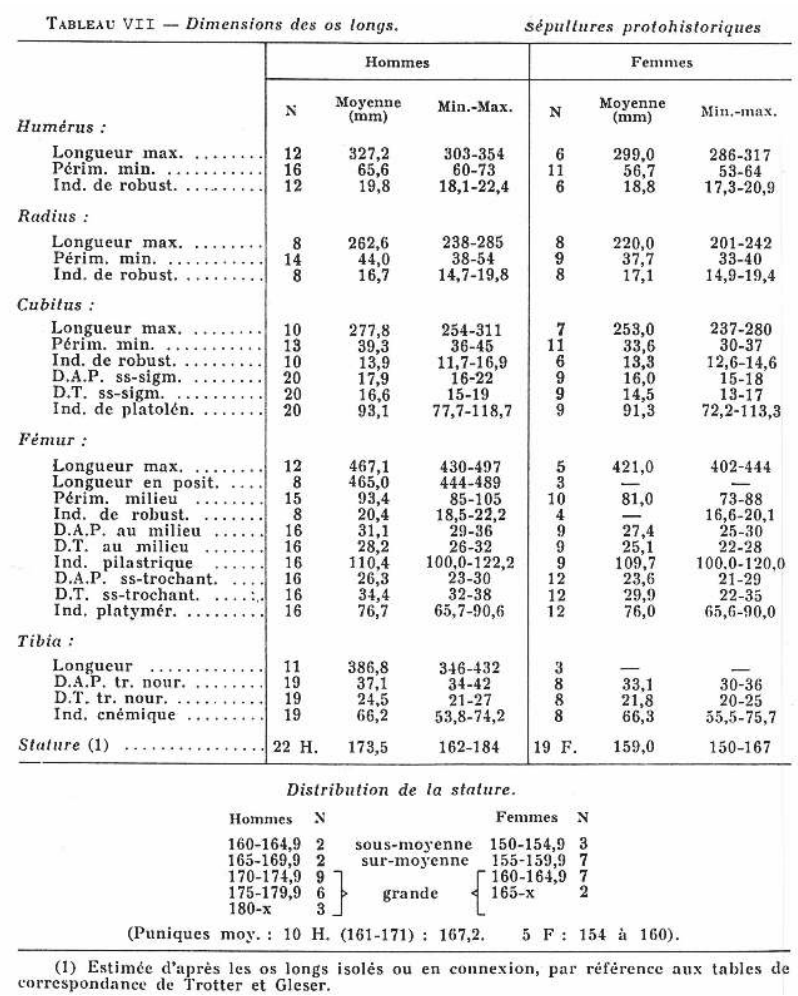

Tableau VIII :- Dimorphisme sexuel.

TABlead VIII- Dimorphisme sexuel.

Différences absolues (en $\mathrm{mm}$ ) et indice (1).

\begin{tabular}{|c|c|c|c|c|c|c|}
\hline \multirow[b]{2}{*}{ Crâxe } & \multicolumn{2}{|c|}{ Sépult. protoh. } & \multicolumn{2}{|c|}{ Sépult. puniques } & \multirow[b]{2}{*}{$\begin{array}{l}\text { Algériens } \\
\text { actuels } \\
(2) \\
\text { Indice }\end{array}$} & \multirow[b]{2}{*}{$\begin{array}{l}\text { Egyptiens } \\
\text { (3) } \\
\text { Indice }\end{array}$} \\
\hline & $\begin{array}{c}\text { Différ. } \\
\text { (en } \\
\text { faveur } \\
\text { des } \\
\text { hommes) }\end{array}$ & $\begin{array}{l}\text { Indice } \\
\text { du } \\
\text { dimor- } \\
\text { phisme }\end{array}$ & Diffẻr. & Indice & & \\
\hline $\begin{array}{l}\text { Longueur } \ldots \ldots \cdots \\
\text { Largeur } \ldots \ldots \cdots \\
\text { Haut. basion } \ldots \ldots \cdots \\
\text { Larg. min. front. } \\
\text { Périm. horiz. } \ldots \cdots \\
\text { Larg. mastoïde } \\
\text { Haut. arc. zyg. } \cdots \cdots \\
\text { Haut. sup. face } \\
\text { Larg. bizygom. }\end{array}$ & $\begin{array}{r}8,01 \\
3,06 \\
5,37 \\
2,16 \\
17,90 \\
4,04 \\
1,43 \\
2,80 \\
9,87\end{array}$ & $\begin{array}{l}104,4 \\
102,2 \\
104,0 \\
102,2 \\
103,5 \\
123,4 \\
122,3 \\
104,0 \\
108,0\end{array}$ & $\begin{array}{r}11,82 \\
4,50 \\
6,45 \\
4,59 \\
28,30 \\
7,24 \\
1,14 \\
7,00 \\
12,21\end{array}$ & $\begin{array}{l}106,6 \\
103,3 \\
105,0 \\
104,8 \\
105,6 \\
143,0 \\
116,4 \\
110,7 \\
109,9\end{array}$ & $\begin{array}{l}105,7 \\
103,7 \\
105,3 \\
104,2 \\
105,3 \\
\overline{-} \\
108,0 \\
106,5\end{array}$ & $\begin{array}{l}104,7 \\
102,5 \\
103,8 \\
102,7 \\
103,8 \\
\overline{-} \\
\overline{105,5} \\
106,5\end{array}$ \\
\hline $\begin{array}{c}\text { Moyenne } \quad \cdots \cdots \cdots \\
\text { Mandiblie }\end{array}$ & & 108,2 & \multirow{4}{*}{\multicolumn{2}{|c|}{$\begin{array}{l}\text { Echantillon } \\
\text { féminin } \\
\text { trop restreint }\end{array}$}} & \multirow{4}{*}{$\begin{array}{c}\text { Echantillon } \\
\text { féminin } \\
\text { trop restreint }\end{array}$} & 104,2 \\
\hline $\begin{array}{l}\text { Longueur ........ } \\
\text { Larg. bicond. } \ldots \ldots \\
\text { Larg. bigoniaque } \\
\text { Haut. br. hor. (M1-M2). } \\
\text { Ep. br. (M1-M2) } \ldots \\
\text { Haut. br. mont. } \ldots \\
\text { Larg. br. mont. } \ldots \ldots \\
\text { Moyenne .......... }\end{array}$ & $\begin{array}{r}4,17 \\
9,50 \\
10,33 \\
3,05 \\
0,42 \\
7,71 \\
2,77\end{array}$ & $\begin{array}{l}104,1 \\
108,4 \\
111,3 \\
111,7 \\
102,7 \\
113,5 \\
108,9 \\
108,6\end{array}$ & & & & $\begin{array}{l}107,5 \\
105,7 \\
109,5 \\
111,5 \\
113,4 \\
107,5 \\
109,1\end{array}$ \\
\hline Moyenne...$\ldots \ldots$ & & 108,6 & & & & 109,1 \\
\hline Stature & 145 & 109,1 & & & & \\
\hline
\end{tabular}

(1) Différences calculées sur les moyennes. Indice du dimorphisme sexuel : $\frac{\text { valour moy. hommes } \times 100}{\text { valeur moy. femmes }}$

(2) D’après F. Demoulin. Le cràne des Algériens.

(3) D'après H. DE Villiens. The skull of the South African Negroe. Johannesburg, 1968. 


\section{Comparaison entre les séries protohistorique, punique et romaine. Analyse typologique (M.-C. Chamla)}

D'après l'étude qui précède, on peut dégager certaines observations qui montrent à la fois une similitude fondamentale entre les séries protohistorique, punique et romaine si l'on tient compte des résultats d'ensemble et d'une diversité qu'une analyse plus fine fait apparaître.

En effet, en se basant sur les moyennes des dimensions absolues du crâne, la comparaison par le test « $t$ » des différentes séries ne révèle pas de différences significatives au seuil de $5 \%$, sauf pour un ou deux traits. S'il existe une diversité, elle n'apparaît que très peu dans les moyennes.

En revanche, les différences apparaissent au sein de chaque série quand on tient compte des catégories majoritaires correspondant aux indices du crâne que nous avons pris comme base de référence.

Celles-ci ne sont pas toujours similaires et le polymorphisme qu'elles révèlent n'est pas toujours comparable entre les trois séries. Certains traits sont plus variables, d'autres le sont moins selon les groupes.

31 L'étendue de la variabilité individuelle à l'intérieur des séries protohistorique et punique a été étudiée à l'aide de l'analyse typologique. Celle-ci a été faite sur la base de 6 caractères métriques (indices crânien, de hauteur-longueur au porion, facial supérieur, orbitaire, nasal, prognathisme) et d'un caractère descriptif (robustesse) et concerne 81 crânes, assez bien conservés pour qu'un signalement satisfaisant de leurs caractères crâniens et faciaux puisse être effectué. On trouvera dans le tableau IX le détail des signalements individuels de ces crânes.

Tableau IX : - Comparaison entre les Algériens des époques protohistorique et romaine et les Puniques. Signification de la différence entre les moyennes par le test t (Hommes).

TAвLEAT IX : - Comparaison entre les Algeriens des époques protohistorique et romaine et les Puniques. Signification de la différence entre les moyennes par le test $t$ (Hommes).

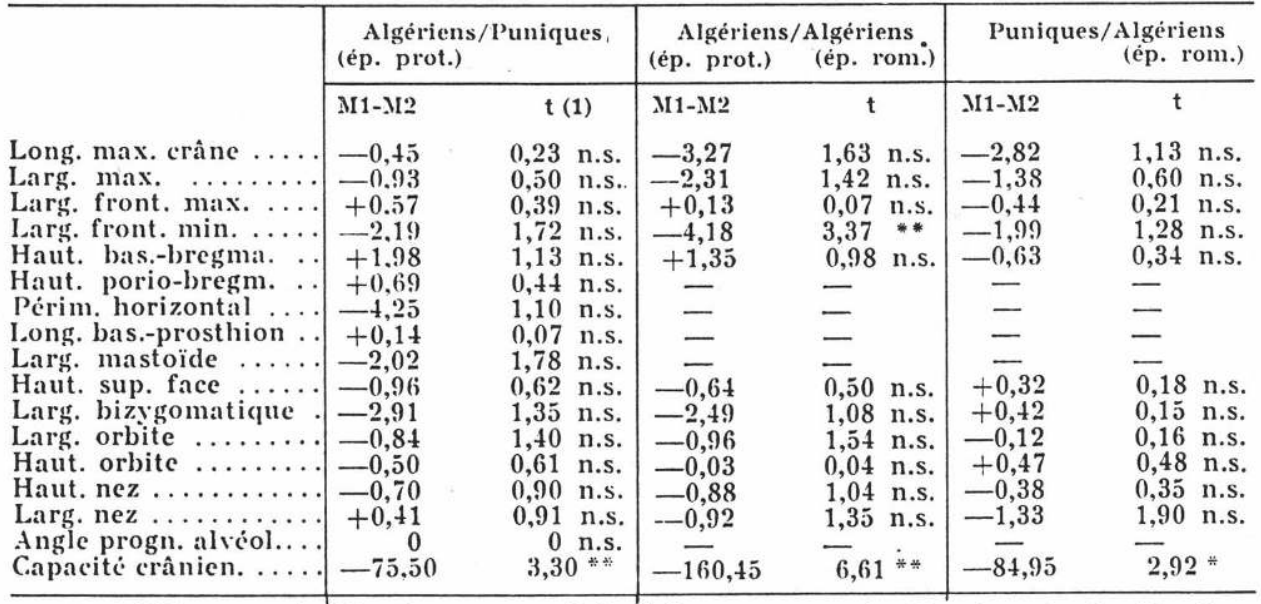

(1) Différences significatives au seuil de $5 \%$ : n.s., non significative; *, significative; *, hautement significative.

32 L'examen de ce tableau confirme l'existence d'un polymorphisme particulièrement accentué. La variabilité individuelle est très grande dans les caractère crâniens et surtout faciaux (indice nasal et prognathisme alvéolaire). Presque aucun crâne ne 
possède le même signalement. Aussi, un regroupement préalable est-il nécessaire si l'on veut dégager les principaux types morphologiques de ces populations. Nous avons montré (1973) que des différences dans le rapport cranio-facial constituent un des traits les plus frappants de la morphologie des Algériens actuels. Ce rapport représente d'ailleurs classiquement un élément important dans l'appréciation des types morphologiques. Un regroupement des sujets a été effectué sur cette base et est indiqué dans le tableau XI avec l'indication des tendances majoritaires des cinq autres caractéristiques crâniennes et faciales.

Trois éléments majeurs se détachent de la douzaine de types crânio-faciaux qui se sont trouvés regroupés. Ce sont les suivants :

a) Des dolichocrânes à face longue (22\%) dont les autres caractères sont une robustesse variable, une voûte de hauteur moyenne, des orbites de hauteur moyenne, un nez étroit à moyen, une tendance au prognathisme.

b) Des mésocrânes à face de hauteur moyenne (21 \%), dont les autres caractères montrent les mêmes tendances que les précédents, mais qui sont plus fréquemment orthognathes.

c) Des dolichocrânes à face de hauteur moyenne (16\%) dont les autres caractères montrent les mêmes tendances que le type $a$, sauf une moindre fréquence du type robuste.

Les 33 crânes restants, soit plus de $40 \%$ de la série totale, montrent une grande diversité du rapport crânio-facial.

A quels types raciaux peut-on rattacher ces divers éléments?

Les dolicho-hyperdolichocrânes à face longue ou moyenne, ainsi que les mésocrânes à face moyenne, ou plus courte, semblent pouvoir être rattachés au type méditerranéen, les uns, la majorité, moyennement robustes et graciles, les autres plus robustes. Le petit nombre de mésocrânes à face longue peut être rattaché à la variante atlantoméditerranéenne. Le prognathisme ou la platyrhinie de certains d'entre eux, non associés, pourraient être éventuellement considérés comme résultant d'un métissage avec un élément négroïde dont un petit nombre de représentants existe dans les séries protohistorique et punique, comme on le verra plus loin, mais on sait qu'une certaine tendance au prognathisme peut exister chez les Méditerranéens en dehors de toute influence mélanoderme.

Tableau X - Signalement des hommes et des femmes des sépultures protohistoriques, puniques et romaines (catégories majoritaires à partir de $25 \%$ de sujets) (1).

\begin{tabular}{|c|c|c|c|c|c|c|}
\hline \multirow[b]{3}{*}{ 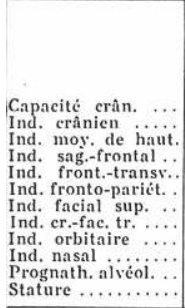 } & \multicolumn{3}{|c|}{ Iommes } & \multicolumn{3}{|c|}{ Femmes } \\
\hline & Sép. prohist. & Sép. romaines & Sép. puniques & Sép. prohist. & Sép. romaines & Sép. puniques \\
\hline & 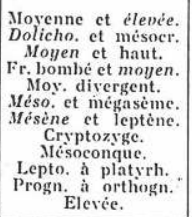 & 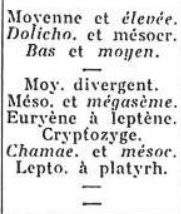 & $\begin{array}{l}\text { Moovenne et élevie. } \\
\text { Dolicho. et mésocr. } \\
\text { Moyen. } \\
\text { Fr. moyen. } \\
\text { Moy. divergent. } \\
\text { Méso. et mégenseme. } \\
\text { Mésène et leptène. } \\
\text { Cryptozyge. } \\
\text { Chamae. et mésoc. } \\
\text { Lepto. et mésors. } \\
\text { Progn. et mésogn. } \\
\text { Sur-moyenne. }\end{array}$ & 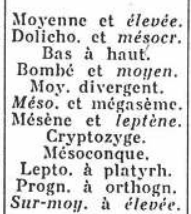 & 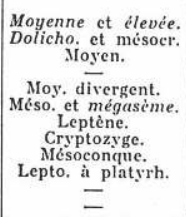 & 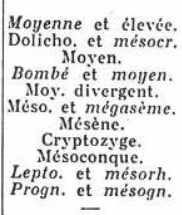 \\
\hline
\end{tabular}

(1) En ilalique les catégories qui rassemblent le plus grand nombre de sujets. 
Tableau XI - Classement des crânes regroupés selon le rapport cranio-facial (Protohistoriques et Puniques).

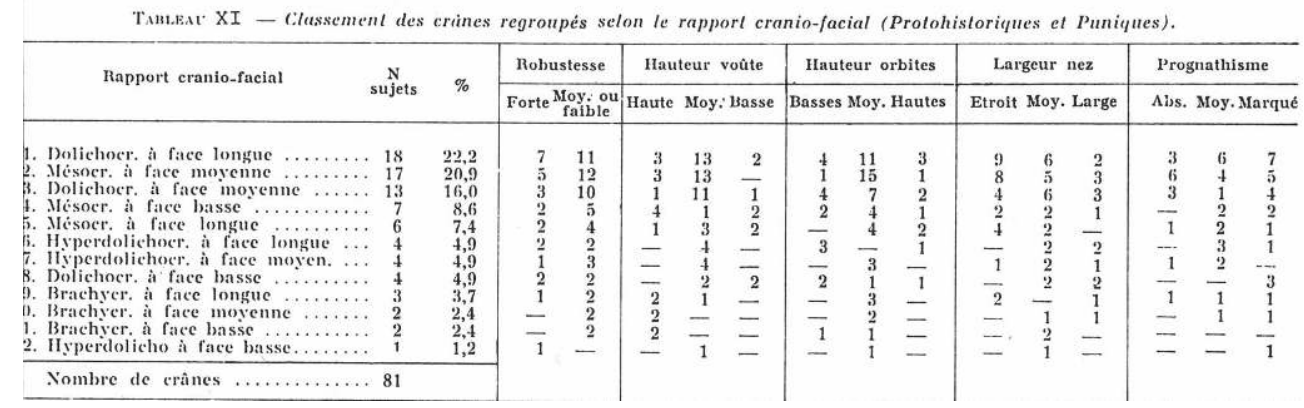

Les autres éléments minoritaires sont des brachycéphales ( 7 sujets) dont la face est de longueur variable. Certains de ceux à face longue pourraient être éventuellement considérés comme des dinaroïdes, ceux à la face moyenne ou courte comme des alpinoïdes.

Enfin, il ne faut pas négliger l'incidence de l'élément mechtoïde dont les traits typiques ont été retrouvés sur certains crânes ainsi que de l'élément négroïde.

\section{L'élément mecthtoïde}

On peut isoler 6 crânes, 5 dans la région orientale de l'Algérie et un à Carthage, présentant des caractères mecthtoïdes typiques, c'est-à-dire grande robustesse, arcades sus-orbitaires développées, gonions extroversés, os nasaux saillants, face courte. Ce sont les sujets suivants: Beni Messous (2.03), Sila (3.94, 3.96, 3.98), Roknia (3.17), Carthage (4.33). On note toutefois chez les Beni Messous une face longue et chez Sila (3.98) l'existence de caractères très mechtoïdes sur la face et une absence d'extroversion goniaque, mais ces traits se rencontrent également dans la série mechtoïde d'Afalou. (Ajoutons à titre indicatif que la calotte de Maktar - Tunisie - non incluse dans l'analyse typologique en raison de son état incomplet, très mechtoïde, possède des bourrelets sus-orbitaires remarquables et une grande épaisseur de parois. A signaler également, dans la série romaine étudiée par Chabeuf, un crâne de Sila et un de Tindja à caractères mechtoïdes.) 


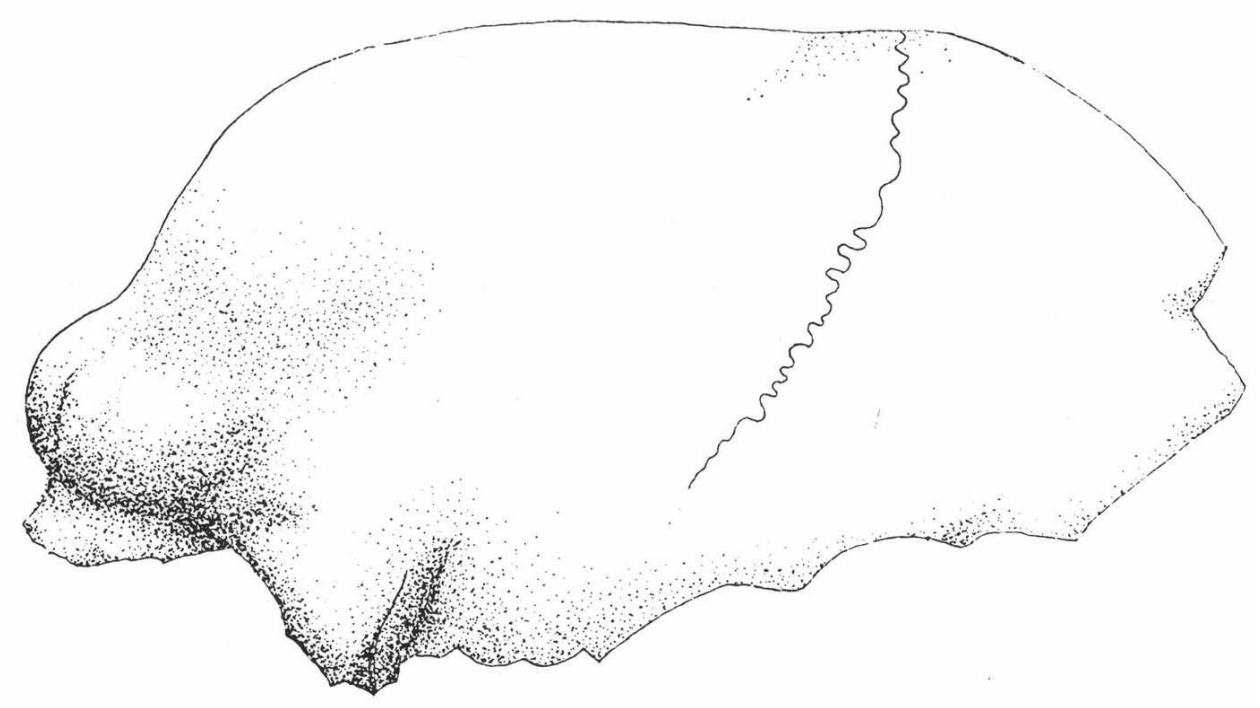

\section{L'élément négroïde}

Sur certains crânes, on note une platyrhinie plus ou moins accentuée associée à un prognathisme plus ou moins marqué. Ce sont là des traits que l'on pourrait considérer comme négroïdes. Si l'on se base uniquement sur l'association de ces deux traits, dix crânes pourraient être considérés comme négroïdes. Certains sont typiques, comme le crâne 3.52 de Gastel qui possède une gouttière sous-nasale, des os nasaux aplatis, un prognathisme facial et alvéolaire accentué, un menton effacé, ainsi que la femme de Djelfa (2.11) dont la face, bien qu'étroite et longue, est fortement prognathe avec un rebord sous-nasal en gouttière, des os nasaux aplatis et, trait culturel répandu chez les Mélanodermes d'Afrique, un limage d'une incisive supérieure. D'autres sont moins typiquement négroïdes, mais peuvent être cependant considérés comme tels, ce sont les crânes de Beidj (2.10), Tiddis (5.02), Roknia (3.05 et 3.37), Gastel (3.54), Sigus (coll. Thomas 3.79), Carthage (4.27 et 4.36).

Compte tenu de ces éléments d'information, on peut effectuer un second classement des crânes permettant de les rattacher à des types raciaux définis (tableau $X$ ).

\section{Position anthropologique des Protohistoriques algériens et des Puniques parmi les populations anciennes du Bassin méditerranéen}

Il existe de nombreuses données, publiées dans la littérature sur les populations postnéolithiques et du début des temps historiques du pourtour de la Méditerranée. Certaines reposent sur des échantillons suffisants, tandis que d'autres sont malheureusement trop fragmentaires et difficilement utilisables statistiquement. Si nous avons pu recueillir dans l'ensemble des séries suffisantes pour l'Afrique septentrionale et l'Europe, il n'en est pas de même pour le Proche-Orient sur lequel il existe, à notre connaissance, très peu de publications concernant des séries 
importantes datant des périodes post-néolithiques. La majorité des études, qui sont nombreuses, concernent de très faibles séries.

Tableau XII - Classement des crânes protohistoriques et puniques selon leur appartenance raciale.

Tanteac XII -- C:lassement des crines prolohisloriques el puniques selon leur appartenance raciale.

\begin{tabular}{|c|c|c|c|c|c|c|}
\hline \multirow[b]{2}{*}{ Elément méditerranéen: } & \multicolumn{2}{|c|}{$\begin{array}{l}\text { Protohist. } \\
\text { d'Algérie }\end{array}$} & \multicolumn{2}{|c|}{ Puniques } & \multicolumn{2}{|c|}{ Total } \\
\hline & $x$ & $\%$ & $x$ & $\%$ & $x$ & $\%$ \\
\hline $\begin{array}{l}\text { Hyperdolicho-dolichocr. à face longue... } \\
\text { Hyperdolicho-dolichocr. à face moyenne. } \\
\text { Hyperdolicho-dolichocr. à face basse.... } \\
\text { Mésocrânes à face longue (atlanto-médit.) } \\
\text { Mésocrânes à face moyenne.............. } \\
\text { Mésocrànes à face basse................. }\end{array}$ & $\begin{array}{r}14 \\
10 \\
1 \\
4 \\
9 \\
3\end{array}$ & $\begin{array}{r}24,56 \\
17,54 \\
1,75 \\
7,01 \\
15,78 \\
5,26\end{array}$ & $\begin{array}{l}4 \\
5 \\
1 \\
2 \\
5 \\
\\
2\end{array}$ & $\begin{array}{r}16,66 \\
20,83 \\
4,16 \\
8,33 \\
20,83 \\
8,33\end{array}$ & $\begin{array}{r}18 \\
15 \\
2 \\
6 \\
14 \\
5\end{array}$ & $\begin{array}{r}22,22 \\
18,51 \\
2,46 \\
7,40 \\
17,28 \\
6,17\end{array}$ \\
\hline $\begin{array}{l}\text { Total Méditerranéens sensu lato.......... } \\
\text { Element brachycéphale: }\end{array}$ & 41 & 71,92 & 19 & 79,16 & 60 & 74,07 \\
\hline $\begin{array}{l}\text { A face longue (dinaroïde ?) } \ldots \ldots \ldots \ldots \ldots \\
\text { A face movenne (alpinoïde } \text { ?) } \ldots \ldots \ldots \ldots \\
\text { A face basse (alpinoïde ?) } \ldots \ldots \ldots \ldots\end{array}$ & $\frac{2}{1}$ & $\frac{3,50}{1,75}$ & $\overline{1}$ & $\begin{array}{l}-4,16 \\
4,16\end{array}$ & $\begin{array}{l}2 \\
1 \\
2\end{array}$ & $\begin{array}{l}2,46 \\
1,23 \\
2,46\end{array}$ \\
\hline Total des éléments brachỵcéphales...... & 3 & 5,26 & 2 & 8,33 & 5 & 6,17 \\
\hline Element mechtoïde & 5 & 8,77 & 1 & 4,16 & 6 & 7,40 \\
\hline $\begin{array}{r}\text { Elément négroïde } \ldots . \\
\text { Total } \ldots\end{array}$ & $\begin{array}{r}8 \\
57\end{array}$ & 14,03 & $\begin{array}{r}2 \\
24\end{array}$ & 8,33 & $\begin{array}{l}10 \\
81\end{array}$ & 12,34 \\
\hline
\end{tabular}

41 Au total, 31 séries originaires des principaux pays d'Afrique septentrionale, d'Europe méridionale et du Proche-Orient et s'échelonnant de l'âge du Bronze à l'époque romaine, ont pu être rassemblées.

Les séries féminines étant moins nombreuses que les séries masculines, nous avons limité nos comparaisons à ces dernières.

La distance globale entre ces groupes et les protohistoriques et Puniques d'Afrique du nord a été évaluée à l'aide $\mathrm{du}_{2} \mathrm{H}$ de Penrose (1954) à partir des dimensions suivantes, en principe non corrélées - ou faiblement - entre elles : longueur, largeur, hauteur basi-bregmatique (ou porio-bregmatique) du crâne, hauteur supérieure de la face, largeur bizygomatique, largeur du nez, longueur basion-prosthion, cette dernière mesure permettant d'évaluer le prognathisme alvéolaire dont la mesure directe est rarement indiquée dans la littérature.

(Tableau XIII et XIV pages suivantes.) 
Comparaison entre les Protohistoriques algériens et des séries anciennes du Bassin méditerranéen. Matérialisation graphique des différences « $d$ » entre les moyennes exprimées en unités d'écart-type commun d'après la formule de Penrose. Les distances moyennes les plus faibles sont seules indiquées.

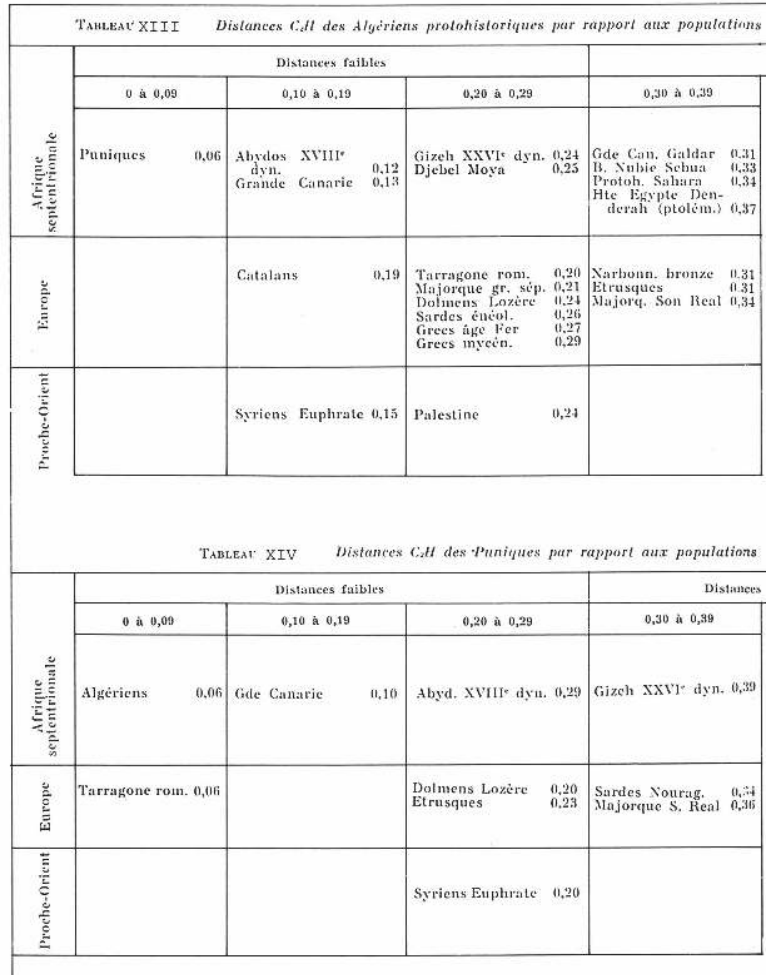

anciennes dn Bassin méditerranten, classies par ordre de yrundeut eroissante.

\begin{tabular}{|c|c|c|c|c|}
\hline moyemnes & & Distances. appreciables & & \\
\hline 0,40 a 0,19 & 0,50 \& 0,59 & 4,64 a $0.7 \%$ & 0,70 iो 0,90 & \\
\hline Denderah rom. $\quad 0,45$ & & & Moy. Eң. Siwa & 0.85 \\
\hline 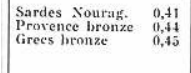 & & & $\begin{array}{l}\text { Minorque } \\
\text { Bas-Languedoc } \\
\text { Valence } \\
\text { Chypre }\end{array}$ & $\begin{array}{l}0,73 \\
0,73 \\
0,74 \\
0,78\end{array}$ \\
\hline $\begin{array}{ll}\text { Tures cuiver } & 0.42 \\
\text { Mésopot. Kish } & 0,45\end{array}$ & & & & \\
\hline
\end{tabular}

anciennes du Bassin méditerranéen, classées par ordre de grandeur croissante.

\begin{tabular}{|c|c|c|c|}
\hline moyenues & & Distances appréciables & \\
\hline $0,40 \AA 0,49$ & $0,50 \& 0,59$ & 0,60 is 0,70 & $0,70 \backsim 0,90$ \\
\hline $\begin{array}{ll}\text { ide Can Galdar } & 0,41 \\
\text { i) jebel Nooya } & 0,+6\end{array}$ & $\begin{array}{l}\text { Denderah ptolém. } 0.51 \\
\text { Soluna } \\
\text { Denderah rom. } \\
0,56,59\end{array}$ & Protoh. Sahara $\quad 0.64$ & Siwa \\
\hline 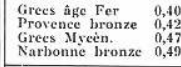 & Grees bronze $\quad 0,55$ & Bas-Langucdoc $\quad 0,62$ & $\begin{array}{ll}\text { Minorque } & 0.81 \\
\text { Chypre } & 0.81 \\
\text { Valence néo-énéo. } & 0.80 \\
1,00\end{array}$ \\
\hline atestine & & $\begin{array}{ll}\text { Tures cuivre } & 0,64 \\
\text { Mésop. Kish } & 0,69\end{array}$ & \\
\hline
\end{tabular}




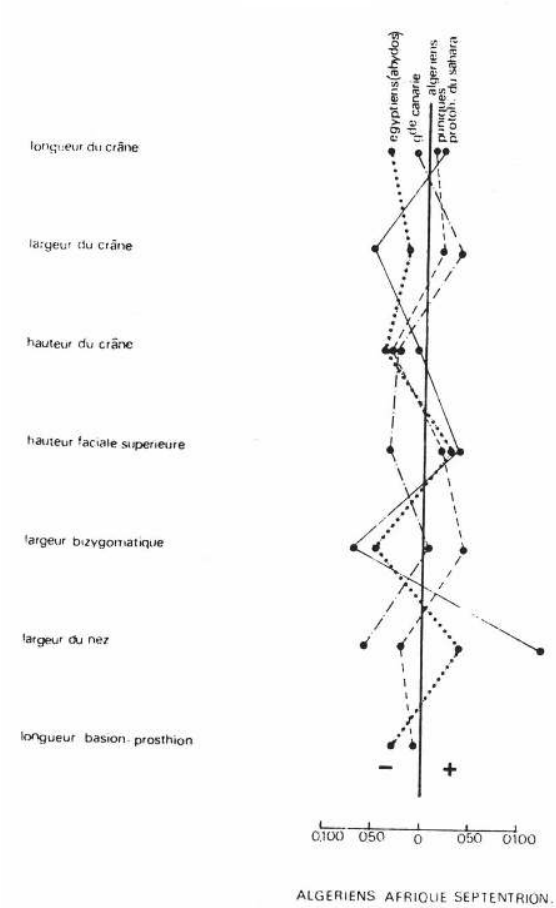

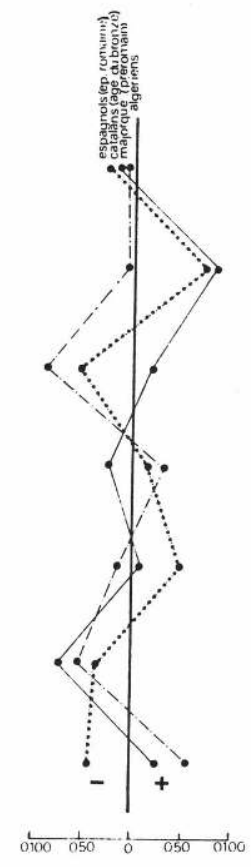

ALGERIENS EUROPE

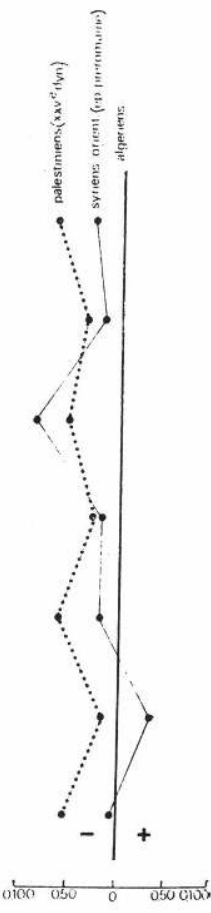

ALCGEAIENS PROCHE-ORIENI
44

Ces distances, classées par ordre de grandeur croissante et par grandes régions géographiques, sont indiquées dans les tableaux XI et XII.

L'examen de ces tableaux permet quelques remarques intéressantes. Si les Algériens et les Puniques s'orientent davantage vers les populations de la Méditerranée occidentale, on constate néanmoins des affinités avec deux séries proche-orientales du premier millénaire avant J.-C. En particulier, la distance est assez faible avec la série syrienne de l'Euphrate. Vis-à-vis des populations protohistoriques du Sahara central et méridional, les distances sont relativement élevées.

Parmi les populations de la Méditerranée occidentale, les séries du nord de l'Espagne et des îles de la Méditerranée occidentale (Catalogne, et Tarragone, Majorque, Sardaigne énéolithique) sont plus proches des Nord-Africains que la série du sud de l'Espagne (Valence). Parmi les populations d'Afrique septentrionale, deux séries égyptiennes, Abydos du II millénaire (Haute Égypte) et Gizeh du $\mathrm{I}^{\mathrm{er}}$ millénaire (Moyenne Égypte), ainsi que la série générale des Canariens, préhispaniques de Grande Canarie (et non celle du tumulus de Gaïdar), paraissent assez proches des Algériens des sépultures protohistoriques. Les affinités des Puniques apparaissent moins regroupées.

47 A partir de ces indications, nous avons repris les séries les plus proches des Algériens et des Puniques et évalué la différence entre les moyennes par le test « $t$ » de Student, en y ajoutant trois autres dimensions (largeur minimale du front, hauteur de l'orbite et périmètre horizontal).

48 Une restriction s'impose néanmoins dans l'interprétation des résultats du test « $t$ " dont la valeur augmente avec l'étendue de la série. C'est ainsi que la série très importante de la Grande Canarie offre des différences presque toutes significatives visà-vis des Algériens, alors que des différences absolues plus fortes vis-à-vis des Puniques ne sont pas significatives du fait du nombre restreint de ces derniers. Il en est de même pour les résultats concernant la faible série de Majorque. 
Si l'on considère les différences les plus faibles, les affinités des Algériens protohistoriques avec les populations du nord de l'Espagne de l'âge du Bronze et de Majorque semblent se confirmer. Leur crâne est cependant moins large que chez les Catalans, plus long et moins haut, ils offrent donc une moindre tendance à la mésocéphalie que ces derniers. Leur face est également un peu longue et plus étroite. Vis-à-vis de la série mayorcaine, la voûte crânienne des Algériens est aussi beaucoup moins élevée, mais de longueur et de largeur analogues, leur face est moins longue et un peu plus large.

50 Le rapprochement avec les Syriens orientaux suggéré par le $\mathrm{C}_{2} \mathrm{H}$ apparaît également se confirmer, mais les Algériens ont un crâne plus volumineux, plus long, plus large, et surtout beaucoup plus haut, leur face est plus longue et plus large. Cependant, les différences ne sont vraiment marquées que pour la hauteur de la voûte.

1 Les Puniques paraissent se rapprocher davantage de la série espagnole romaine de Tarragone qui leur est plus ou moins contemporaine, bien qu'on relève des différences assez importantes portant sur la longueur et la largeur du crâne.

Au total, la position anthropologique des Protohistoriques algériens et des Puniques vis-à-vis des populations du Bassin méditerranéen s'accorde assez bien avec leur situation géographique. Situés à mi-chemin entre les pays de la Méditerranée occidentale et le nord-est de l'Afrique, ils offrent des affinités avec les anciens habitants de l'Espagne du nord et de l'Égypte. D'autre part, dans la mesure où chez les anciens habitants de la Syrie orientale le type méditerranéen prédominait, il n'est pas étonnant de constater des ressemblances entre ces derniers et les Algériens protohistoriques où prédominait ce type morphologique. Mais il ne faut pas oublier non plus que les Algériens de l'époque protohistorique étaient des descendants des Protoméditerranéens capsiens et néolithiques. Les hommes qui apportèrent la culture capsienne en Afrique du nord ont vraisemblablement une origine proche-orientale. Les individus de type robuste dolicho- et mésocéphales de grande taille des sépultures protohistoriques pourraient bien en être les descendants, tandis que les individus plus graciles se rapprocheraient du type protoméditerranéen occidental gracile que l'on trouve déjà à l'époque néolithique en Algérie et en Tunisie. (Tableaux XV et XVI page suivante.)

Tableau XV - Comparaison entre les Algériens et les populations du Bassin méditerranéen les plus proches définies pat le C.II Signification de la différence entre les moyennes par le test $t$.

\begin{tabular}{|c|c|c|c|c|c|c|c|c|c|c|c|c|c|c|}
\hline \multirow{2}{*}{$x$} & \multicolumn{2}{|c|}{$\begin{array}{c}\begin{array}{c}\text { Algeriens/ } \\
\text { Abydos }\end{array} \\
\end{array}$} & \multicolumn{2}{|c|}{$\begin{array}{c}\text { Algériens/ } \\
\text { Gr. Canarie }\end{array}$} & \multicolumn{2}{|c|}{$\begin{array}{c}\text { Algériens/ } \\
\text { Catalans }\end{array}$} & \multicolumn{2}{|c|}{$\begin{array}{l}\text { Algeriens/ } \\
\text { Tarragone }\end{array}$} & \multicolumn{2}{|c|}{$\begin{array}{l}\text { Algériens/ } \\
\text { Majorque }\end{array}$} & \multicolumn{2}{|c|}{$\begin{array}{c}\text { Algériens/ } \\
\text { Syriens }\end{array}$} & \multicolumn{2}{|c|}{$\begin{array}{c}\text { Algériens/ } \\
\text { Palestiniens }\end{array}$} \\
\hline & M1-N2 & $t(1)$ & M1-M2 & $t$ & M1-M12 & $t$ & M1-M2 & $t$ & M1-M2 & $t$ & M1-M2 & $t$ & M1-M2 & $t$ \\
\hline 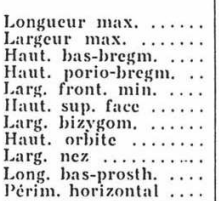 & $\begin{array}{r}+2,56 \\
+0,86 \\
+1,98 \\
\overline{-} \\
+0,96 \\
+2,62 \\
-0,72 \\
+1,70 \\
+1,50\end{array}$ & 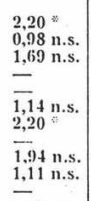 & $\begin{array}{l}+0,77 \\
-1,71 \\
+2,01 \\
-2,11 \\
+1,11 \\
+0,25 \\
+1,05 \\
+1,03 \\
=\end{array}$ & 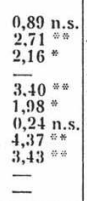 & $\begin{array}{l}+1,27 \\
-3,91 \\
-0,82 \\
-0,71 \\
+0,99 \\
-0,55 \\
+1,75 \\
+1,43 \\
-0,60\end{array}$ & $\begin{array}{l}0,87 \text { n.s. } \\
3,72 * \text { *. } \\
0,66 \text { n.s. } \\
0,56 \text { n.s. } \\
0,78 \text { n.s. } \\
0,27 \text { n.s. } \\
1,29 \text { n.s. } \\
2,86 \% \\
0,08 \text { n.s. }\end{array}$ & $\begin{array}{l}+\mathbf{1}, 87 \\
-3,31 \\
+3,01 \\
-1,12 \\
-0,31 \\
-0,71 \\
-2,71 \\
-0,85 \\
+0,10 \\
+0.63 \\
+2,15 \\
-6,90\end{array}$ & 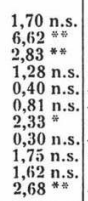 & $\begin{array}{l}+0,55 \\
+0,40 \\
+4,32 \\
+1,70 \\
-1,38 \\
-1,27 \\
+0,73 \\
+0,13 \\
+0,93 \\
-1,00\end{array}$ & $\begin{array}{ll}0,32 & \text { n.s. } \\
0.27 & \text { n.s. } \\
2,08 & \text { n.s. } \\
1,22 & \text { n.s. } \\
1,09 & \text { n.s. } \\
0.85 & \text {..s. } \\
0,36 & \text { n.s. } \\
0,19 & \text { n.s. } \\
1,50 & \text { n.s. } \\
0,18 & \text { n.s. }\end{array}$ & $\begin{array}{l}+1,94 \\
+1,04 \\
+5,16 \\
+7,19 \\
+0,54 \\
+0,88 \\
+1,30 \\
-0,20 \\
-0,0,5 \\
+0,04 \\
+1,05\end{array}$ & $\begin{array}{l}1,35 \text { n.s. } \\
0,74 \text { n.s. } \\
3,46 * * . \\
5,70 * * \\
0,48 \text { n.s. } \\
0.69 \text { n.s. } \\
0,78 \text { n.s. } \\
0,37 \text { n.s. } \\
1.10 \text { n.s. } \\
0,02 \text { n.s. } \\
0,27 \text { n.s. }\end{array}$ & $\begin{array}{l}+4,17 \\
+1,89 \\
+2,81 \\
+\overline{0,99} \\
+1,09 \\
+3,5,5 \\
+0,65 \\
+0,33 \\
+2,95 \\
+5,40\end{array}$ & 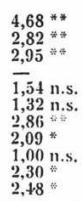 \\
\hline
\end{tabular}

(1) Différences signifieatives au seuil de $5 \%$ : n.s., non significative; ", significative; "*, hautement significative. 
Tableau XVI. - Comparaison entre les Puniques et les populations du Bassin méditerranéen les plus proches définies par le C.II. Signification de la différence entre les moyennes par le test $t$.

Tableat XVI. - Comparaison entre les Puniques et les populations du Bassin médilerraneen les plus proches définies par le G.all. Signification de la différence entre les moyennes par le test $t$.

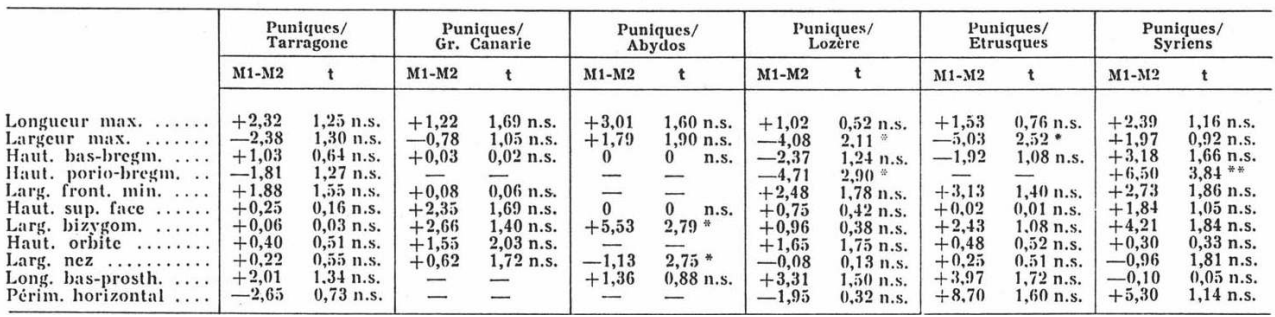

\section{BIBLIOGRAPHIE}

Protohistoire Antiquité (M.-C. Chamla)

ANGEL J., « Population size and microevolution in Greece », Cold Spring Harbor symposia on quantitative biology, 1951, t. 15, p. 343-351.

BATRAWI A., « The racial history of Egypt and Nubia. Part I, The craniology of Lower Nubia from Predynastic times to the sixth century A.D. ", J. Roy anthr. Inst. 1945, t. 75, p. 81-101.

BERTHERAND Dr., et BOURJOT Dr., « Fouilles des dolmens du plateau de Beni Messous accompagnées de déductions anthropologiques du Dr Bourjot », Bull. Soc. algér. climatol, 1986, t. 5, p. 88-101.

BERTHIER A., « Les Bazinas de Tiddis », Libyca, 1956, t. 4, p. 147-153.

BERTHOLON L., « Note sur deux crânes phéniciens trouvés en Tunisie », L’Anthropologie, 1890, p. 314-319.

ID, « Documents anthropologiques sur les Phéniciens », Bull. Soc. d'Anthrop. Lyon, 1892. Id, « Étude comparée sur des crânes de Carthaginois d'il y a 2400 ans et de Tunisois contemporains ", Rev. tunisienne, 1911.

ID. et CHANTRE C, Recherches anthropologiques dans la Berbérie orientale (Tripolitaine, Tunisie, Algérie), Lyon, 1913, 2 vol. BERTRAND A., « Fouilles de Roknia », Bull. Soc, d'Anthrop., 1868, p. 628.

BOEV P., Die Rassentypen der Balkanhalbinseln, Bulgar, Akademie der Wissensch, Sofia, 1972, 269 p. BOISREDON L. de, « Les tombeaux circulaires du Djebel Mistiri », Rec. notes et mém. Soc. archéol. Constant., 1873, t. 16, p. 70-76.

BOULINIER G. et CHABEUF M., "Les squelettes romains et paléochrétiens du musée d'Alger », Bull, et Mém. Soc. d'Anthrop., 1971, nº 1, p. 7-43.

BOURGUIGNAT J., Histoire des monuments mégalithiques de Roknia, Paris, 1868.

BOURJOT Dr., « Fouilles de la Société », Bull. Soc. algér. climatol, 1869, t. 6, appendice. 
ID., « La grotte du Grand Rocher de Guyotville, Alger », Matér. Hist. Natur. de l'Homme, 1869, t. 5, p. 448-450.

BRUNON « Mémoire sur les fouilles exécutées au Madras'en mausolée des rois de Numidie ",Rec. des Notes et Mém. Soc. archéol. de Constantine, 1873-1874, t. 16, p. 303-350.

CAMPS G., « Les dolmens de Beni Messous », Libyca, 1953, t. 1, p. 329-372.

Id., « La céramique des sépultures berbères de Tiddis », Libyca, 1956, t. 4, p. 155-203.

ID., « Données nouvelles sur les tombeaux du Djebel Mistiri, d'après une note de M. Latapie », Libyca, 1958-1959, t. 6-7, p. 229-242.

ID., « Relations protohistoriques entre la Berbérie orientale et les îles italiennes », Congr. Préhistor. de France, $16^{\mathrm{e}}$ session, Monaco, 1959 (1965), p. 329-337.

ID., Aux origines de la Berbérie. Monuments et rites funéraires protohistoriques. Paris, Arts et Métiers graphiques, 1961, $628 \mathrm{p}$.

ID., « A propos d'une étude sur la protohistoire de la Tunisie », Libyca, 1963, t. 11, p. 295-306.

ID.et H. CAMPS-FABRER, « La nécropole mégalithique du Djebel Mazela à Bou Nouara », Mém. du CRAPE, Alger, 1964, 89 p.

ID., « Une date absolue de monument funéraire protohistorique : le tumulus de l'oued Montana (Ferkane) », Lybica, 1964, t. 12, p. 298-299.

ID., « Essai de classification des monuments protohistoriques de l'Afrique du nord », Bull. Soc. préhis. franc., 1965, t. 42, fasc. 2, p. 476-481.

ID., « Notes de Protohistoire nord-africaine et saharienne, v. Dates absolues concernant la protohistoire du Maghreb et du Sahara ", Libyca, 1970, t. 18, p. 235-239.

ID., « Formation des populations méditerranéennes de l'Afrique du nord », Coll. intern. Soc. Biologie humaine, 1968, Hammamet, Tunisie (1970), p. 51-57.

ID., Les civilisations préhistoriques de l'Afrique du nord et du Sahara, Paris, Doin, 1974, 366 p.

CANTACUZÈNE G., « Contribution à la craniologie des Etrusques ", L’Anthropologie, 1909, p. 329-352.

CARTON Dr., « Découvertes épigraphiques et archéologiques faites en Tunisie », Mém. Soc. Sciences de Lille, 1895, 428 p. (Teboursouk et Dougga, p. 326-344).

ID., « Notice sur Dougga, les fouilles du Dar-el-Acheb », Rec. Notes et Mém. Soc. archéol. Constantine, 1898 , t. 32, p. 210-241.

CHABASSIÈRE J., « Ruines et dolmens du Fortas et de ses contreforts », Rec. Notes et Mém. Soc. archéol. Constantine, 1886-1887, t. 24, p. 124 et 127 (Sigus).

CHAMLA M.-C, « Les populations anciennes du Sahara et des régions limitrophes. Études des restes osseux humains néolithiques et protohistoriques. Alger ", Mém. du CRAPE, n 9, 1968, 245 p.

CHAMLA M.-C, « Les hommes des sépultures protohistoriques et puniques d'Afrique du nord (Algérie et Tunisie) », L'Anthropologie, 1975, t. 79, nº 4, p. 659-692 et 1976, t. 80, p. 75-116. CINTAS P., «Éléments d'études pour une protohistoire de la Tunisie. Université de Tunis, Faculté des Lettres ", Archéologie, Préhistoire, t. 7, P.U.F., 1961, 170 p.

COLLIGNON R., «Crânes de la nécropole phénicienne de Mahédia (Tunisie) ». L’Anthropologie, 1892, p. $163-173$. 
DASTUGUE J., « Crânes protohistoriques trépanés ou pathologiques d'Afrique du Nord », L'Anthropologie, 1973, n 1-2, p. 63-92.

DELATTRE R.P., « Fouilles exécutées dans la nécropole punique voisine de Sainte-Monique », C.R. Académie Inscript, et Belles Lettres, 1901, p. 583.

DEMOULIN F., Le crâne des Algériens, thèse fac. des Sciences, Paris, 1972.

DERRY D., « A study of crania from the oasis of Siwah », Harv. African studies, 1927, t. 8, p. 201-222.

FAIDHERBE L., « Recherches anthropologiques sur les tombeaux mégalithiques de Roknia », Bull.

Académie d'Hippone, 1867, t. 4, p. 1-76.

ID., « Nécropole mégalithique de Mazela sur la route de Constantine à Guelma », Bull. Acad.

Hippone, 1868, t. 6, p. 63-65 (Bou Nouara).

ID., « Nécropole mégalithique de Mazela », Matér, pour l'Histoire de l’Homme, 1869, t. 5, p. 222.

ID., « Fouilles dans les dolmens de Tébessa et de Guestel », Bull. Soc. d'Anthrop., 1869, p. 543 (Djebel Osmor).

ID., « Les dolmens d'Afrique », Congr. d'Anthrop. et d'Archéol. préhistor., Bruxelles, 1872, p. 406-420. FÉRAUD L., " Monuments dits celtiques de la province de Constantine », Réc. Notes et Mém. Soc. archéol. Constantine, 1863, t. 8, p. 214-234 (Ras-el-Aïn-Bou Merzoug).

FUSTÉ ARA M., « Estudio sobre unos craneos de la cultura Helinistico-romana de Baleares », Trab Inst. Bernard, Sahagun, 1950, t. 11, p. 7-46.

ID., « Craneos de la edad del Bronce procedentes de una cueva sepulcral de Son Maymo en Petra (Mallorca)», Trab. Inst. Bernard Sahagun, 1953, t. 13, n 3, p. 153-171.

ID., Estudio antropologico de los pobladores neo-eneoliticos de la region valenciana, Servie, de investig. prehistorica, Valence, $1957, \mathrm{n}^{\circ} 20,128 \mathrm{p}$.

ID., Estudio antropologico de los esqueletos inhumados en tumulos de la région de Gaïdar (Gran Canaria), Las Palmas, 1961-1962, 122 p.

GARRALDA M., « Restos humanos hallados en el poblado de Almallotx (Escorca, Mallorca) », Trabaj. de Antrop., 1971, t. 16, n 2, p. 63-71.

ID., « Restos humanos pertenencientes al Bronce antiguo de Mallorca », Trabajos de Antrop., 1972, t. $16, n^{\circ} 3$, p. $123-141$.

ID., « Estudio entropologico de la cueva de Son Bosc. Aportación al conocimiento del Hierro de Mallorca », Trabajos de Antrop., 1973, t. 16, n 4, p. 229-241.

GREBENART D., « Sépultures protohistoriques de la région de Ferkane », Libyca, 1961-1962, p. 171-195.

HAMY E., « Cités et nécropoles berbères de l'Enfida », Bull, géogr. et histor., 1904.

HANNEZO M., « Note sur des sépultures phéniciennes découvertes près de Mahédia (Tunisie) », L'Anthropologie, 1892, p. 161-162.

ICARD « Notes sur les dolmens de Dougga », Bull. Soc. d'archéo. deSousse, 1905, t. 3, p. 253-256.

JACQUOT L., « La nécropole dolménique de Roknia », Rec. Notes, Mém. Soc. archéol. Constantine, 1916, p. 207.

KовELT W., « Les dolmens de Guyotville », Rev. Ethnogr., 1887, p. 133-149. 
LE DÛ R., « Les tombeaux ronds du Djebel Mistiri », IVe congrés fédér. Soc. savantes Afr. du nord, 1938, p. 565-587.

LETHIELLEUX J., « Vestiges préhistoriques et protohistoriques de la région de Djelfa », Libyca, 1965, t. 13 , p. 249-265.

LETOURneau et PAPILlaUlt, « Crânes des dolmens du Madracen près de Batna », Bull. Soc, d'Anthrop., 1896, p. 347.

LOGEART F., " Grottes funéraires, hypogées et caveaux sous roche de Sila », Réc. Notes, Mém. Soc. archéol. Constantine, 1935-1936, t. 63, p. 69-105.

MARCHAND H., « Nouveaux documents anthropologiques et zoologiques recueillis aux dolmens de Beni Messous ", Bull. Soc. Hist. nat. Afrique du nord, 1930, t. 21, p. 135-146.

ID., Mélanges d'anthropologie et de sociologie nord-africaine, Alger, 1951, 237 p. (Beni Messous).

MARTIGNAT M. de, « Note sur une station mégalithique de Djelfa », Bull. Acad. d'Hippone, 1914-1921, t. 24, p. $129-133$.

MAXIA C, « Sull'Antropologia dei Protosardi, nota III. I ritrovamenti eneolitici della grotta "Su cungiareddu de Serafini” (Carbonia) », Rendic. del Semin. della fac. de Scienze, Univ. Cagliari, 1963 , t. $33, n^{\circ} 1-2$, p. $1-21$.

ID et ATZENI E., « La necropoli eneolitica di S. Benedetto di Iglesias », Atti della 8'et 9'reun. scient. dell'Instit. italiano de preistoria et protoistoria, Florence, 1964, p. 123-135.

MUKHERJEE R., RAO C, TREVOR J., The ancient inhabitants of Djebel Moya, Cambridge Univ. Press, 1955 , $123 \mathrm{p}$.

PAUPHILET D., « Monuments mégalithiques à Maktar », Karthago, 1953, t. 4, p. 49-83.

PEARSON K., DAVIN A., « On the biometric constants of human skull », Biometrika, 1924, t. 16 , p. 328-368.

PEE-LABORDE L., Anthropologie préhistorique des Grands Causses, Montpellier, 1962, 68 p.

PENROSE L., « Distance, size and shape », Annals of Eugenics, 1954, t. 18, p. 337-343.

PINCHON Dr., « Stations de surface et tumuli de la région de Bossuet et du Telagh (Oran) », Cong. préhist. de France, $12^{e}$ session, Toulouse, 1936, p. 375-402.

PONS J., « Restos humanos procedentes de las necropolis de Tarragona y Ampurias (Gerona), Trabajos Inst. Bernard de Sahagun, 1951, t. 7, p. 19-206.

ID., « Craneos de epoca romana procedentes de la necropolis de Son Taxequet (Mallorca), Trabajos Inst. Bernard de Sahagun, 1951, t. 12, p. 9-22.

PRUNER-BEY, « Études sur les crânes de Roknia », Matér. Hist. prim. et natur. de l'Homme, 1869, t. 5, p. 202-204.

QuATrefages A. de, et hamy E., Crania ethnica, Paris, 1882.

REBOUD Dr, « Note pour servir à l'étude de la nécropole de Sigus », C.R. X congrès AFAS, Alger, 1881, p. $1142-1149$.

REYGASSE M., Monuments funéraires préislamiques de l'Afrique du nord, Paris, 1950, 128 p.

RIALLE G. de, « Allées couvertes d'Ellez (Tunisie) (communication de documents découverts par M. Poinsot) », Bull. Soc. d'Anthrop., 1884, t. 7, p. 366-371. 
RIQUET R., Anthropologie du Néolithique et du Bronze ancien, Poitiers, Texier, 1970, 279 p.

RISDON R., « A study of the cranial and other human remains from Palestine excavated at Tell Duweir (Lachish)», Biometrika, 1939, t. 31, p. 99-166.

ROFFO Dr., « Sépultures indigènes antéislamiques en pierres sèches, étude de trois nécropoles de l'Algérie centrale », Rev. Africaine, 1938, t. 32, p. 197-242. Suivi d'une notice de Leblanc : «Étude des ossements humains des sépultures de l'Ourek, de Tamda et d'Aïn el Hamara (Algérie centrale).

INDEX

Mots-clés : Préhistoire, Anthropologie, Sahara, Protohistoire, Maghreb, Origines, Histoire contemporaine 